\title{
Thalamic connections of the core auditory cortex and rostral supratemporal plane in the macaque monkey
}

\author{
Brian H. Scott ${ }^{1,{ }^{*}, \text { Kadharbatcha S. Saleem }}{ }^{1}$, Yukiko Kikuchi ${ }^{1,2}$, Makoto Fukushima $^{1,3}$, \\ Mortimer Mishkin ${ }^{1}$, and Richard C. Saunders ${ }^{1}$ \\ ${ }^{1}$ Laboratory of Neuropsychology, National Institute of Mental Health, National Institutes of Health \\ (NIMH/NIH), Bethesda, MD 20892, USA \\ 2Present address: Institute of Neuroscience, Newcastle University Medical School, Newcastle \\ Upon Tyne, NE2 4HH, UK \\ ${ }^{3}$ Present address: RIKEN Brain Science Institute, 2-1 Hirosawa, Wako City, Saitama \\ 351-0198,Japan
}

\begin{abstract}
In the primate auditory cortex, information flows serially in the mediolateral dimension from core, to belt, to parabelt. In the caudorostral dimension, stepwise serial projections convey information through the primary, rostral, and rostrotemporal (AI, R, and RT) core areas on the supratemporal plane (STP), continuing to the rostrotemporal polar area (RTp) and adjacent auditory-related areas of the rostral superior temporal gyrus (STGr) and temporal pole. In addition to this cascade of corticocortical connections, the auditory cortex receives parallel thalamocortical projections from the medial geniculate nucleus (MGN). Previous studies have examined the projections from MGN to auditory cortex, but most have focused on the caudal core areas AI and R. In this study, we investigated the full extent of connections between MGN and AI, R, RT, RTp, and STGr using retrograde and anterograde anatomical tracers. Both AI and R received nearly $90 \%$ of their thalamic inputs from the ventral subdivision of the MGN (MGv; the primary/lemniscal auditory pathway). By contrast, RT received only 45\% from MGv, and an equal share from the dorsal subdivision (MGd). Area RTp received $\sim 25 \%$ of its inputs from MGv, but received additional inputs from multisensory areas outside the MGN (30\% in RTp versus 1-5\% in core areas). The MGN input to RTp distinguished this rostral extension of auditory cortex from the adjacent auditory-related cortex of the STGr, which received $80 \%$ of its thalamic input from multisensory nuclei (primarily medial pulvinar). Anterograde tracers identified complementary descending connections by which highly processed auditory information may modulate thalamocortical inputs.
\end{abstract}

\footnotetext{
"Corresponding author Brian H. Scott, Ph.D., Laboratory of Neuropsychology, National Institute of Mental Health, NIH, Building 49, Room 1B80, Bethesda, MD 20892, Phone (301) 443-7867, Fax (301) 402-00467, brianscott@ mail.nih.gov; bhscott@outlook.com. The authors declare no conflicts of interest.

All authors had full access to all the data in the study and take responsibility for the integrity of the data and the accuracy of the data analysis. Study concept and design: RCS, MM, BHS, MF, KS. Acquisition of data: BHS, YK, RCS, MF. Analysis and interpretation of data: BHS, YK, KS. Drafting of the manuscript: BHS. Critical revision of the manuscript for important intellectual content: RCS, KS. Obtained funding: MM. Study supervision: RCS.
} 


\section{Graphical Abstract}
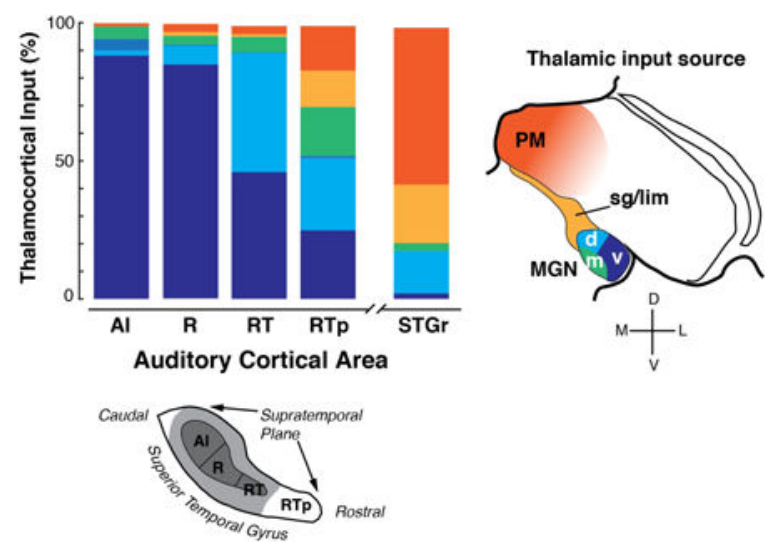

\section{Keywords}

rhesus; primate; medial geniculate; pulvinar; thalamus; corticothalamic; RRID: AB_10013220;

RRID: AB_1502299; RRID: AB_2536200; RRID: AB_477329

\section{Introduction}

The flow of information in the auditory cortex of primates diverges along two pathways proposed to serve distinct functional roles: a dorsal stream processing spatial aspects of sound sources, and a ventral stream processing sound quality (Rauschecker, 1998; Romanski et al., 1999; Rauschecker and Scott, 2009). Functional evidence indicates that the auditory ventral stream in macaque monkeys includes the entirety of the supratemporal plane (STP) and adjacent superior temporal gyrus (STG), extending rostrally into the dorsal temporal pole (Poremba et al., 2003; Poremba et al., 2004; Petkov et al., 2008; Kikuchi et al., 2010; Ng et al., 2013; Fukushima et al., 2014; Scott et al., 2014). Mapping the ventral stream, and the route by which auditory information reaches the rostral auditory areas, is fundamental to understanding the neural basis of sound perception.

The predominant model of auditory cortex in the macaque consists of a central core surrounded by a belt of secondary areas on the STP, flanked laterally by a parabelt region on the STG (Fig. 1; (Hackett et al., 1998a; Kaas and Hackett, 1998; de la Mothe et al., 2006a; Hackett, 2011; de la Mothe et al., 2012a). The auditory cortex is defined as those regions receiving significant input from the medial geniculate nucleus $(\mathrm{MGN})^{1}$, the thalamic relay for auditory information ascending from the inferior colliculus (IC). The primary (or 'lemniscal') pathway from the central IC passes through the ventral division of the MGN (the MGv), which in turn projects to the core regions. The belt and parabelt regions receive input from the dorsal division of the MGN (MGd) but little or no input from MGv ((Hackett et al., 1998b; Jones, 2007; Hackett, 2011). In the mediolateral direction, information flows in a predominantly serial progression from core to belt, and from belt to parabelt (Galaburda

${ }^{1}$ Also known as the medial geniculate body (MGB) or complex (MGC); we use MGN for parallelism to the lateral geniculate nucleus (LGN) of the visual system. 
and Pandya, 1983; Hackett et al., 1998a; Hackett, 2011), with comparatively sparse direct connections from core to parabelt (Hackett et al., 1998a; Scott et al., 2017). In the orthogonal direction, information flows caudorostrally within the core, belt, and parabelt (Galaburda and Pandya, 1983; Morel and Kaas, 1992; Morel et al., 1993; Jones et al., 1995; de la Mothe et al., 2006a; Scott et al., 2017).

The core region in macaques forms an elongated strip extending caudo-rostrally along the STP, and is subdivided into three areas: the primary auditory cortex AI, the rostral area R, and the rostrotemporal area RT. A defining characteristic of the core is dense immunostaining for parvalbumin (Jones et al., 1995; Hackett et al., 1998a; Scott et al., 2017), a feature shared with the MGv that projects to it (Molinari et al., 1995; Jones, 2007). Responses of neurons in the core are typically sharply tuned for frequency, and the borders between $\mathrm{AI}$ and R, and between R and RT, are indicated by reversals in the tonotopic gradient (Merzenich and Brugge, 1973; Recanzone et al., 2000; Petkov et al., 2006; Bendor and Wang, 2008; Scott et al., 2011; Fukushima et al., 2012). Rostral to RT, a continuation of weakened parvalbumin immunoreactivity on the STP has been recently proposed to constitute a distinct rostro-temporal polar area (RTp; (Saleem et al., 2007; Scott et al., 2017). The areas of the core and rostral STP differ in their temporal response properties, such that neurons in R, RT, and RTp exhibit increasingly longer and more variable response latencies, and less precise temporal encoding of modulated stimuli, than neurons in AI (Recanzone et al., 2000; Bendor and Wang, 2008; Kikuchi et al., 2010; Scott et al., 2011; Camalier et al., 2012).

We recently described the corticocortical connections by which auditory information flows caudorostrally from AI to R, R to RT, RT to RTp, and from RTp to the rostral STG and dorsal temporal pole (STGr/TGdg; (Scott et al., 2017). This predominantly stepwise connectivity is consistent with a hierarchical processing model, which would predict the increase in response latency beyond AI. However, corticocortical connections do not tell the full story, as each area also receives parallel thalamic inputs from the MGN and other nuclei. Functional differences among the core areas may arise from intracortical processing, but because the subdivisions of the MGN differ in their physiological properties (Preuss and Muller-Preuss, 1990; Edeline et al., 1999; Jones, 2007; Bartlett and Wang, 2011; Bartlett, 2013), such differences may also be inherited from distinct thalamic inputs. The thalamic connections of the caudal STP areas that feed into the auditory dorsal stream have been carefully quantified (Hackett et al., 2007; Smiley et al., 2007), but few data are available for the ventral stream areas of the STP, particularly those rostral to area R (Burton and Jones, 1976; Molinari et al., 1995; Rauschecker et al., 1997).

Here we describe the thalamic connections of the core auditory areas (AI, R, and RT), RTp, and STGr/TGdg as revealed by a series of sixteen anatomical tracer injections spanning the caudorostral extent of the STP (Fig. 1). Each area is characterized by a distinct distribution of thalamic inputs from the subdivisions of the MGN and from other auditory-related nuclei. In addition, the use of anterograde tracers identified corticothalamic projections to the MGN from all areas, elaborating upon the complex recurrent network evident in the corticocortical connections of the auditory ventral stream (Scott et al., 2017). 


\section{Materials and Methods}

\section{Subjects}

Five adult rhesus monkeys (Macaca mulatta, four males, 5-10 years old) weighing between 5.5 and $13 \mathrm{~kg}$ were used. All procedures adhered to the Guide for the Care and Use of Laboratory Animals (National Research Council), and were carried out under a protocol approved by the Institutional Animal Care and Use Committee of the NIMH. Four of the five animals (OR, VA, CC, SP) were prepared with a complete commissurotomy in a separate surgery prior to tracer injection, allowing the left and right hemispheres to serve as independent cases. In the remaining animal (MQ) the commissures were intact, and only one hemisphere was injected (only ipsilateral connections are presented). Each case is indicated by a two-letter animal designation followed by ' 1 ' or ' $r$ ' to indicate the injected hemisphere (e.g., OR-r; see Table 1). For all figures, data are displayed in a right-hemisphere orientation.

\section{Tracers}

Fifteen tracer injections were placed in the core auditory areas (AI, R, RT) and RTp, spanning approximately $15 \mathrm{~mm}$ of the STP (Table 1; Fig. 1). An additional tracer injection was placed in the STGr lateral to RTp. Twelve of these cases were also included in a previous publication that described cortico-cortical connectivity within the temporal lobe (Scott et al., 2017). We used retrograde tracers Fast blue (FB), Diamidino yellow (DY), and cholera toxin subunit $\beta$ (CTB), and bi-directional tracers Fluoro-ruby (FR; dextranconjugated tetramethylrhodamine), Fluoro-emerald (FE; dextran-conjugated fluorescein), and Biotinylated dextran amine (BDA). The volume, concentration, and supplier of the tracers for each injection are indicated in Table 1 (all were injected in aqueous solutions). Although BDA (10 kDa molecular weight) is primarily an anterograde tracer, some cells were labeled by retrograde transport (Fig. 11). To optimize bi-directional transport of FR, the $10 \mathrm{kDa}$ and $3 \mathrm{kDa}$ molecular weights were mixed in equal parts. However, FE reliably produced bi-directional transport using only the $10 \mathrm{kDa}$ molecular weight.

\section{Surgery and Injections}

Prior to each surgery, the animal was sedated with ketamine $(10 \mathrm{mg} / \mathrm{kg})$, intubated, and then maintained at a surgical level of anesthesia with isofluorane (1-4\%, to effect). Body temperature was maintained with a heating pad, and the head was fixed in a head-holder. Vital signs (heart and respiration rate, temperature, oxygen saturation, and $\mathrm{CO}_{2}$ ) were monitored throughout the procedure, and intravenous fluids were provided. For the commissurotomy, unilateral bone and dural flaps were turned to expose the cerebral midline. With the aid of an operating microscope, the corpus callosum, hippocampal commissure, and anterior commissure were visualized and transected with a glass pipette. The dural flap was then replaced, the bone flap sewn in position, and the wound closed in anatomical layers. A prophylactic dose of analgesics and antibiotics was administered, and continued postoperatively in consultation with the facility veterinarian.

In a separate surgery (at least three months after the commissurotomy), anatomical tracers were injected by direct visualization of the STP, as follows. The fronto-temporal bone and dural flaps were turned to expose the length of the lateral sulcus and STG in one 
hemisphere. In three cases (VA, CC, SP) the banks of the lateral sulcus were carefully separated with fine forceps and a small glass pipette attached to a vacuum pump. This sulcal separation extended as far medially as the fundus of the inferior limb of the circular sulcus, with special care taken to avoid damaging the pial surface of the STP or compromising blood vessels bridging the lips and banks of the sulcus. In a fourth case the surface of the STP was visualized by aspiration of the overlying tissue of the parietal operculum in the right hemisphere (OR-r), and the lateral sulcus of the left hemisphere was opened as described above (OR-1).

Injections were placed at a depth of approximately $1.5 \mathrm{~mm}$ below the pial surface, using a Hamilton syringe with a 30-gauge needle for CTB and dextran tracers, or a 26-gauge needle for fluorescent tracers (FB and DY). Injection sites on the STP were located in relation to gross anatomical landmarks, with the most rostral site (targeting RTp) located about $3 \mathrm{~mm}$ caudal to the temporal pole, and the RT, R, and AI sites spaced at intervals of about $5 \mathrm{~mm}$ (spacing was adjusted to avoid blood vessels). RT and R injections were placed medial to the lip of the circular sulcus when possible, as this typically corresponds to the auditory core region (Jones et al., 1995; Hackett et al., 1998a), whereas the more lateral sites on the STP itself fall within the belt. Injections targeting AI were placed caudal to the posterior end of the circular sulcus, near the center of the STP in the mediolateral dimension, often near a small annectant gyrus associated with the primary area (Jones et al., 1995). After completion of injections in one hemisphere, the dural flap was sutured, the bone flap sewn back into place, and the procedure repeated in the opposite hemisphere, allowing for as many as 10 injections per animal. After replacement of the second bone flap, the wound was closed in anatomical layers. Animals were treated with postoperative antibiotics, analgesics, and dexamethasone $(0.5-1 \mathrm{mg} / \mathrm{kg})$ to reduce brain swelling.

In the remaining case (MQ-r), micro-electrocorticography $(\mu \mathrm{ECoG})$ arrays had been implanted on the surface of the STP (Fukushima et al., 2012; 2014). After the conclusion of the recording experiments the tonotopic reversals between the core fields and RTp were identified, and a site in low-frequency RTp was selected for tracer injection. The arrays on the surface of the STP were visualized by aspiration of the overlying tissue of the parietal operculum. A Hamilton syringe, positioned in a stereotaxic manipulator for a vertical approach to the STP, was lowered into the cortex through a $0.5-\mathrm{mm}$ hole in the array (Fukushima et al. 2012, see their Fig. 1A). After the injection of tracer, the needle was left in place for 10 minutes before being gradually withdrawn.

\section{Histology}

After a survival period of approximately 14 days (range: 13-16 days), animals were deeply anesthetized with pentobarbital and perfused transcardially with $0.5 \mathrm{~L}$ of saline, followed by $0.5 \mathrm{~L}$ of $1 \%$ paraformaldehyde and $8 \mathrm{~L}$ of $4 \%$ paraformaldehyde, both in $0.1 \mathrm{M}$ phosphate buffer ( $\mathrm{pH}$ 7.4) at room temperature. Brains were then removed from the skull, cryoprotected through a series of glycerols (Rosene et al., 1986), blocked in the coronal plane, and frozen in $-80^{\circ} \mathrm{C}$ isopentane. Sections were cut in the coronal plane on a sliding microtome at a thickness of $40 \mu \mathrm{m}$, and sorted into 10 parallel series in each case. Two or three series were immediately mounted on gelatin-coated slides, air-dried, and coverslipped 
with DPX (Sigma-Aldrich) for the examination of fluorescent tracers (FB or DY). Other series were processed immunohistochemically with the Avidin/Biotin immunoperoxidase method for CTB, FR, or FE labeling, or directly with the Avidin/Biotin method for BDA labeling (see below). Remaining series of sections were processed for thionine, acetylcholinesterase (AChE), or immunohistochemically with antibody against parvalbumin (PV) and a nonphosphorylated epitope of the neurofilament protein (recognized by the SMI-32 antibody). These latter stained sections were used to delineate the cyto- and chemoarchitectonic borders between cortical areas on the plotted sections (Scott et al., 2017). The specificity and characterization of antibodies for tracers (CTB, FR, FE) and PV are shown below.

\section{Antibody characterization}

The antibodies against CTB, FR, and FE were raised against CTB subunit B (http:// antibodyregistry.org/AB_10013220), tetramethylrhodamine (http://antibodyregistry.org/ AB_1502299), and fluorescein (http://antibodyregistry.org/AB_2536200), respectively, and the specificity of each antibody was determined by the manufacturer (see Table 2).

The anti-PV antibody (http://antibodyregistry.org/AB_477329) was raised against PV from purified frog muscle and was determined to be specific by immunoblotting (Western blot) and to specifically stain the $12 \mathrm{kDa}$ molecular weight band identified as PV by Ca-binding (Sigma data sheet). Parvalbumin is a calcium-binding protein associated with a subpopulation of inhibitory interneurons containing gamma-aminobutyric acid (Celio, 1986; Hendry et al., 1989). Staining patterns in the current study (see also Scott et al., 2017) accord with previous descriptions of staining patterns in the macaque cortex (Jones et al., 1995; Hackett et al., 1998a; Saleem et al., 2007; Saleem and Logothetis, 2012) and thalamus (Hashikawa et al., 1991; Molinari et al., 1995; Hackett et al., 1998b; Jones, 2007).

\section{Immunohistochemical procedures}

Immunohistochemical (IHC) staining was used to visualize CTB, FR, and FE. Although FR and FE do fluoresce (e.g., Fig. 1 A, C-E, H, and P), IHC staining provided greater sensitivity in identifying axon fibers and synaptic terminals labeled by anterograde transport.

To visualize CTB, sections were rinsed in phosphate buffered saline (1X PBS, pH 7.4), washed for 30 minutes in $0.6 \%$ hydrogen peroxide $\left(\mathrm{H}_{2} \mathrm{O}_{2}\right)$ to inhibit endogenous peroxides, washed in PBS, and then incubated for $2 \mathrm{~h}$ in blocking serum consisting of $0.3 \%$ Triton $\mathrm{X}-100,2 \%$ bovine serum albumin (BSA), and 3.75\% normal rabbit serum in PBS. Tissue was then incubated in the primary antibody solution (anti-CTB added to the blocking serum as shown in the previous step; see Table 2) for 60 hours at $4^{\circ} \mathrm{C}$ with agitation. After several washes in PBS, sections were then incubated in the secondary antibody solution (biotinylated anti-goat IgG added to the same blocking serum solution described above; see Table 2) overnight at $4^{\circ} \mathrm{C}$ with agitation, followed by another wash in PBS. The sections were then processed with the avidin/biotin staining kit (Vector ABC Elite) for 90 minutes at room temperature, after which sections were washed in PBS and placed in a $0.025 \%$ solution of 3,3-diaminobenzidine tetra hydrochloride as chromogen (DAB; Sigma \#D5637). After 10 minutes, approximately $0.0075 \%$ of $\mathrm{H}_{2} \mathrm{O}_{2}$ was added to initiate the staining 
reaction. The DAB reaction was stopped when satisfactory contrast was achieved (usually 1-3 minutes for CTB). After a final rinse in phosphate buffer, sections were mounted on gelatin-coated slides, air-dried, and dehydrated through ascending grades of ethanol concentrations before being cleared in xylenes and coverslipped in DPX.

The IHC process for FR and FE was similar to that for CTB with some modifications. Sections were rinsed in $0.05 \mathrm{M}$ Tris-buffered saline (TBS, pH 7.6), quenched in $0.6 \% \mathrm{H}_{2} \mathrm{O}_{2}$ for 10 minutes, then incubated for $1 \mathrm{~h}$ in normal blocking serum (as described above, but in TBS). Tissue was incubated in the primary antibody solution (anti-FR or anti-FE, see Table 2) for 3 days at $4^{\circ} \mathrm{C}$ with agitation. After rinsing in TBS, sections were incubated in the secondary antibody solution (as above, but in TBS; see Table 2) for $90 \mathrm{~min}$ at room temperature with agitation. After washing in TBS, the avidin/biotin reaction and DAB staining were carried out as described for CTB staining above.

To visualize $\mathrm{PV}$, sections were rinsed (1X PBS, $\mathrm{pH}$ 7.4), quenched in $0.6 \% \mathrm{H}_{2} \mathrm{O}_{2}$ for 60 minutes, washed in PBS, and then incubated for $2 \mathrm{~h}$ in blocking serum (see CTB staining protocol above). Tissue was incubated in the primary antibody solution (anti-PV, see Table 2) for 3 days at $4^{\circ} \mathrm{C}$ with agitation. After rinsing overnight in PBS at room temperature with agitation, sections were incubated in the secondary antibody solution for $90 \mathrm{~min}$ at room temperature with agitation (biotinylated goat anti-mouse $\operatorname{IgG}[\mathrm{H}+\mathrm{L}]$, Vector \#BA-9200). Sections were then washed in PBS, and the avidin/biotin reaction and DAB staining were carried out as described above.

The BDA series was stained using a streptavidin horseradish peroxidase (HRP) procedure. Sections were washed in $0.05 \mathrm{M}$ TBS ( $\mathrm{pH} 7.6$ ), quenched in $\mathrm{H}_{2} \mathrm{O}_{2}$ ( $0.3 \%$ for 30 minutes), washed in TBS again, then incubated in streptavidin-HRP $(0.5 \mu \mathrm{g} / \mathrm{ml}$; Molecular Probes $)$ overnight at $4^{\circ} \mathrm{C}$ with agitation. Sections were then put through consecutive washes in TBS at $\mathrm{pH} 7.6$, and then at $\mathrm{pH} 8.0$, after which sections were placed in a $0.025 \%$ solution of 3,3diaminobenzidine tetra hydrochloride (DAB; Sigma) at $\mathrm{pH}$ 8.0. The DAB reaction was stopped when satisfactory contrast was achieved (typically 1-3 minutes). In case SP, DAB staining was intensified with nickel ammonium sulfate to enhance contrast.

\section{Data Analysis}

Sections were examined with a Zeiss Imager Z. 1 microscope, and images were captured by a Zeiss Axiocam MRc5 or MRm camera. Digital images were adjusted for brightness and contrast using Adobe Photoshop CS. In brightfield images, the background outside the pial surface was masked. Cortical laminae were drawn on photomicrographs by alignment to an adjacent section stained for thionine or SMI-32.

Sections were plotted at a sampling interval of 0.4 or $0.8 \mathrm{~mm}$ using either a Zeiss Axiophot microscope fitted with an MDplot digitizer and software (AccuStage, Shoreview MN) or a Zeiss Imager Z.1 fitted with the Neurolucida system (MBF Bioscience, Williston VT). The outline of the brain surface was traced, and the individual cells retrogradely labeled by different tracers were plotted. For the anterograde labeling, the individual fibers or terminals were not plotted; instead the distribution of labeling was outlined and represented with two relative thresholds of staining intensity (e.g., the shades of gray in Figs. 3, 5, 7, 10, and 11). 
The plotted sections were exported to Adobe Illustrator CS, where the traced outline of the brain surface was aligned with digital images of corresponding adjacent sections stained for thionine and SMI-32. The border between the gray and white matter, cortical layer 4, and the outlines of subcortical structures were traced digitally. Borders between cortical areas and thalamic nuclei were determined by examination of thionine, $\mathrm{PV}, \mathrm{AChE}$, and (for cortex only) SMI-32 staining patterns (for thalamus, see Results, below; for cortex, see Scott et al., 2017). When these borders were drawn on the plotted sections, all tracer label was hidden in the Adobe Illustrator file so as not to bias the placement of the borders. In all figures, the plotted sections were cropped to show only the relevant regions of the temporal lobe or thalamus.

To facilitate comparison among cases, the caudal pole of the MGN in each case was assigned an arbitrary $\mathrm{A} / \mathrm{P}$ coordinate of $+4.0 \mathrm{~mm}$ relative to the interaural axis (based on (Paxinos et al., 2000; Saleem and Logothetis, 2012), and the relative A/P position of other sections was computed from the section thickness $(40 \mu \mathrm{m})$.

\section{Results}

\section{Anatomical subdivisions of the auditory thalamus}

The cyto- and chemo-architecture of the medial geniculate complex and auditory-related nuclei of the thalamus in monkeys have been well described (Jones 2007, Chapters 8.4, 10.1, and 11.2.3; (Morel et al., 1993; Molinari et al., 1995; Hackett et al., 1998b; de la Mothe et al., 2006b; Hackett et al., 2007). The present study employed the same criteria as in those prior reports, described briefly below.

\section{Subdivisions of the MGN}

The MGN includes four divisions: ventral (MGv), magnocellular (or medial; MGm), and a dorsal division that, in primates, can be subdivided into anterodorsal (MGad) and posterodorsal (MGpd; Fig. 2). In thionine-stained sections, the MGv is characterized by medium-sized, darkly staining cells arranged with a high and generally homogeneous packing density (Fig. 2C, E). Medially, the MGm is distinguished by lower packing density and a wider range of cell sizes, with large darkly staining cells being the most prominent (though small cells are also found within this magnocellular division). Cells within the dorsal subdivision were less densely packed than those in MGv, and often paler staining. The distinction between the dorsal subdivisions MGpd and MGad was subtle in cytoarchitecture, but more apparent in PV-immunostained sections. Posteriorly, MGpd was distinguished from MGv by lower cell packing density and an absence of PV+ cells (Fig. 2D). By contrast, cells and neuropil within MGad were PV+, and MGad was more similar to MGv in terms of packing density (Fig. 2F).

The boundary between the ventral and dorsal divisions, and between MGad and MGpd, is often unclear. Previous studies have identified a "transition zone" $(Z)$ between these subdivisions (Molinari et al., 1995; Hackett et al., 2007). In the interest of parsimony, and to establish distinct borders by which to quantify the distribution of labeled cells, we assigned areas dorsal to MGv to either MGpd or MGad. Based on those cases in which the MGad/pd 
distinction was clear (as in Fig. 2), a common standard was applied across all cases: MGd at A/P level +4.0-5.0 (within $1 \mathrm{~mm}$ of the caudal pole) was designated MGpd, and at +5.2-6.2 $\mathrm{mm}$ was designated MGad.

The lateral border between the MGN and the inferior pulvinar (or, in more rostral sections, the LGN) was reliably distinct, being marked by a clear septum (Fig 2B). The medial border, by contrast, was more diffuse (e.g., Fig. 2E), particularly at the dorsomedial aspect of the nucleus where the MGm is continuous with the suprageniculate nucleus (see below).

Moving caudally through a series of coronal sections, the MGv recedes to occupy only a thin crescent at the ventral edge of the nucleus, such that sections through the caudal pole of the MGN typically contain only MGpd (around $+4.0 \mathrm{~mm}$ A/P by the convention applied in our figures).

\section{Auditory-related thalamic nuclei}

Dorsomedial to the MGN, the suprageniculate nucleus ( $\mathrm{Sg}$ ) adjoins the $\mathrm{MGm}$ and contains similarly darkly-staining cells (though not as large as those within MGm). The $\mathrm{Sg}$ is continuous with the limitans nucleus (Lim), a line of darkly staining cells extending dorsomedially from the MGN and delimiting the ventral border of the pulvinar.

Following Jones (2007, p. 1084), who merged the Sg and Lim because of their similar structure and connections, we treat the $\mathrm{Sg} / \mathrm{Lim}$ as a single entity. In the caudal thalamus, the limitans lies below the medial pulvinar (PM), often separated by the white matter of the brachium of the superior colliculus. In more rostral sections the limitans terminates ventromedial to the caudal subdivision of the mediodorsal nucleus (MD), which appears between the PM and the habenula. The MD was evident as a cluster of darkly stained neurons abutting the lighter and less densely packed pulvinar (Fig. 2B). The lateral and medial subdivisions of PM were distinguished by cytoarchitecture and PV immunostaining (Gutierrez et al., 2000); Jones 2007, chapter 10).

\section{Thalamic connections of auditory cortical areas}

The fifteen tracer injections that were placed along the length of the STP resulted in labeled cells and terminals throughout the MGN, as well as other nuclei of the posterior thalamus. The data show a progressive shift in thalamic inputs between the caudal and rostral STP, such that more rostral areas received less input from the MGv, and a greater proportion of input from other MGN subdivisions, and auditory-related thalamic nuclei outside the MGN.

\section{Connections of areas $\mathrm{Al}$ and $\mathrm{R}$}

Data were analyzed from three tracer injections into the primary auditory cortex (AI): one into caudal AI, a second into rostral AI, and a third near the border between AI and R (Fig. 1A-C; Table 1). Tracer injections into AI labeled cells in all subdivisions of the MGN, but predominantly within MGv through the mid-to-rostral extent of the nucleus. The count of retrogradely labeled neurons within each thalamic subdivision is summarized for all injections in Table 4. 
An injection of FR into caudal AI in case OR-r (Figs. 1A and 3) labeled a dense cluster of cell bodies in the MGv, as well as smaller populations of cells in MGpd, MGm, and MGad (Fig. 3B-D). Patches of anterograde label were co-localized with some of these clusters of filled cells, indicating a reciprocal connection. In addition, some filled cells and one patch of strong anterograde label were observed in the medial pulvinar (Fig. 3B, C). An injection of DY in case VA-r that was placed more rostrally within AI labeled cells exclusively within the MGN (Fig. 1B and Fig. 4, red dots). Most of the filled cells were found within the rostral half of the MGv, where they formed dense clusters (Fig. 4D-F). A much smaller number of cells was observed in the MGm, and very few within MGad and MGpd. A third injection, in which bi-directional tracer FE was placed near the AI/R border in case OR-1 (Fig. 1C) produced a strong cluster of anterograde and retrograde label in rostral $\mathrm{MGv}(+5.6 \mathrm{~A} / \mathrm{P})$, with a secondary cluster in MGad. Only a few cells were located in MGm and MGpd (data not shown; see Table 4, case 3).

Three tracer injections into core area R (Fig 1D-F; Table 1) identified strong reciprocal connectivity with the ventral division of the MGN, and weaker connections with the surrounding subdivisions.

An injection of bi-directional tracer FE produced a large but well-confined injection site spanning all cortical layers of central R (Figs. 1E, 5). Filled cells were located throughout the middle to rostral portion of the MGN, and were co-localized with a dark swath of anterogradely labeled fibers and terminals in the MGv (Fig. 5B, and the corresponding photomicrograph in 5D). In addition, a sparse distribution of both cells and labeled fibers and terminals was found in MGpd, MGm, sg, and medial pulvinar (Fig. 5A, B).

A smaller injection of FE confined to the superficial layers of area R in case VA-r (Figs. 1D, $5 \mathrm{E})$ resulted in a much more restricted distribution of anterograde and retrograde labeling in MGv, but not in other thalamic nuclei. A similar distribution of retrograde labeling was observed in another case, in which an injection of DY was restricted to the superficial layers of rostral R in case VA-1 (Fig. 1F; plotting data not shown but see Table 4, case 6).

The number of labeled neurons within each thalamic subdivision was quantified for each AI and $\mathrm{R}$ injection. When the data were pooled across the three injections in each area, the overall distribution of inputs was nearly identical between AI and R. The overwhelming majority of retrogradely labeled cells were observed within the MGv (88.1\% for AI, 85.0\% for R), and the remaining cells were distributed within MGd (6.0\% for AI, 7.1\% for R) and MGm (4.5\% for AI, 3.2\% for R). Within the MGd, inputs to AI were biased toward the anterodorsal subdivision (4.1\% MGad, $1.9 \% \mathrm{MGpd}$ ), whereas inputs to $\mathrm{R}$ were biased toward the posterodorsal subdivision ( $0.4 \% \mathrm{MGad}, 6.8 \% \mathrm{MGpd})$. Only one out of three AI and R injections labeled any cells in the medial pulvinar (see Table 4; $1.1 \%$ AI, $2.9 \% \mathrm{R}$ ). Cases in which bi-directional tracers were used produced evidence of strong corticothalamic projections that generally followed the same anatomical distribution as the retrogradely labeled cells, being most prominent in MGv (e.g., Fig. 5B-D). 


\section{Connections of area RT}

Three injections placed into the rostrotemporal area RT were analyzed (Fig. 1G-I; Table 1), revealing a pattern of thalamocortical inputs distinct from that in $\mathrm{AI}$ and $\mathrm{R}$. Whereas the distribution of labeled cells following injections into the caudal auditory cortex (AI and R) was biased toward the middle to rostral half of the MGv, injections into RT tended to label cells in the caudal half of the MGN, with a more even distribution between the ventral and dorsal subdivisions.

This is exemplified by the case depicted in Figure 4 (case VA-r), in which the distribution of labeled cells following an injection of FB into RT (blue dots) is complementary and largely non-overlapping with that produced by the DY injection into rostral AI (red dots). Filled cells were most abundant near the caudal pole of the MGN, which consists primarily of MGpd (Fig. 4A-B). Cells labeled by the RT and AI injections were intermingled within MGv near the middle of the nucleus (Fig. 4C), but in more rostral sections only a thin array of cells at the ventral edge of MGv was labeled by FB (Fig. 4E- F). A second injection of retrograde tracer (DY) into the rostral portion of area RT in case CC-r replicated this pattern (Figs. 1I, 6), in which labeled cells were dense in MGpd and MGv near the caudal pole (Fig. 6A-B), but restricted to the most ventral edge of MGv more rostrally (Fig. 6C-F); a few scattered cells were found in medial pulvinar in both cases (e.g., Figs. 4C, 6A).

In a third case, a bi-directional tracer injection in RT confirmed the pattern of thalamocortical projections in the prior two cases and revealed complimentary corticothalamic projections. An injection of FR into the rostral portion of RT in case VAl (Figs. 1H, 7) resulted in retrogradely labeled cells within MGpd and the caudal-to-middle portion of MGv, as well as a few cells within MGm and medial pulvinar (Fig. 7A- D). Anterograde label followed a similar pattern, including dense patches of terminals and fibers across MGv and MGpd (e.g. Fig. 7B-C). Rostrally, anterograde label was limited to the most ventral edge of MGv (Fig. 7E-F), the same area occupied by labeled cells in the previous two RT cases. No clear patterns of labeled terminals were evident across injections in the dorso-ventral or medio-lateral axes.

The overall distribution of thalamic inputs to area RT was qualitatively different from that in $\mathrm{AI}$ and R. Whereas AI and R drew nearly $>85 \%$ of their thalamic inputs from MGv, inputs to RT were split evenly between MGv and MGpd (46.0\% and 43.2\%, respectively), with the remainder arising mostly from the MGm (5.6\%) or medial pulvinar (2.9\%).

\section{Connections of area RTp}

Data from six tracer injections into the rostral STP were analyzed (Fig. 1J-M, O-P; Table 1). These experiments demonstrated that although the rostrotemporal-polar area RTp receives input from the ventral and dorsal MGN, a greater proportion of thalamocortical input arises from the MGm, Sg/Lim nucleus, and medial pulvinar than was evident following injections into AI, R, or RT.

In case OR-1 (Fig. 1J, 8) and injection of retrograde tracer DY was located near the rostral end of RT or the caudal end of RTp. Because the injection itself obscured the cyto- and chemo-architecture in the adjacent sections, this site could not be placed into RT or RTp 
with sufficient confidence, so it was designated as 'RT/RTp border' (and excluded from quantitative comparisons between areas). As in the three RT cases described above, labeled cells were predominantly found in the caudal half of the MGN, with their distribution focused mainly in MGpd but not in MGad (compare Fig. 8B-D vs. E-G). The few labeled cells in the rostral MGN were restricted to the ventral edge of the nucleus (Fig. 8F-G). In distinction to those RT cases, labeled cells in caudal MGN were clearly densest in the MGv (Fig. 8C-D), followed by the MGm (Fig. 8D-E). Another distinct feature of this case, relative to the RT injections, is the constellation of filled cells extending dorsomedially from the MGm through the Sg/Lim nucleus (Fig. 8B-D), into the medial pulvinar (Fig. 8A-D). As will be seen in cases described below, this pattern of connections is consistent across RTp injections.

An injection of retrograde tracer CTB into area RTp in case MQ-r (Figs. 1M, 9) substantially replicated the case above. Most labeled cells were distributed in MGv, MGpd, and MGm (Fig. 9B-D), but the proportion of cells in MGv was lower than in the previous case (Table 4). In rostral MGN, filled cells were distributed in a thin layer following the ventral edge of the nucleus, falling within both MGv and MGm (Fig. 9F-G). Outside the MGN, labeled cells were found throughout the $\mathrm{Sg} / \mathrm{Lim}$ nucleus as well as the most caudal regions of the medial pulvinar (Fig. 9A-C). In addition, a few labeled cells were located in the caudal portion of the mediodorsal nucleus (Fig. 9D), to a greater extent than was seen following AI, R, or RT injections.

The thalamocortical projections observed in the prior two RTp injections were complemented by descending corticothalamic projections, as demonstrated by three additional injections of bi-directional or anterograde tracers. In case VA-r (Figs. 1P, 10), an injection of FR into rostral RTp replicated the distribution of retrogradely labeled neurons previously described, and also identified dense fields of fibers and axon terminals within MGpd and, to a lesser extent, MGv (Fig. 10A-B). Sparser regions of anterograde label were found within MGm, MGad, and the medial pulvinar (Fig. 10C-D), as well as the mediodorsal nucleus of the thalamus (Fig. 10E). Rostrally, fibers and terminals formed a thin layer at the ventromedial edge of the MGN (Fig. 10E-F), the same location occupied by filled cells in the cases described above (e.g., Figs. 8F-G, 9F-G). The descending projections from area RTp to MGpd, MGv, MGm, and medial pulvinar were confirmed by BDA injections in cases OR-1 (Figs. 10, 11A-G) and SP-r (Figs. 1K, 11H-I).

The combined retrograde data from five RTp cases (Table 4; excluding data from the RT/RTp border) indicate that about $25 \%$ of thalamic inputs to RTp arose from the MGv and $26 \%$ from MGpd. The remaining half arose in roughly equal proportion from MGm (17.7\%), Sg/Lim (13.2\%), and PM (16.1\%).

\section{Connections of STGr/TGdg}

To directly compare the projections from thalamus to the rostral STP and adjacent STG, a retrograde tracer injection was placed lateral to RTp on the gyrus at the border between the rostral STG (STGr) and the dorsal temporal pole (TGdg; Figs. 1N, 12). Labeled cells were found throughout the medial pulvinar and within the $\mathrm{Sg} / \mathrm{Lim}$ nucleus, as well as in MGpd (Fig. 12A-C). In contrast, very few cells were located in MGm and MGv (Fig. 12C). This 
finding suggests that the thalamic connections of area RTp are distinct from those of the adjacent STGr/TGdg, which derives the majority of its input (56.8\%) from the medial pulvinar and only $20 \%$ from the MGN (Table 4).

\section{Discussion}

A series of tracer injections along the STP revealed a systematic caudal-to-rostral shift in the distribution of thalamic inputs to AI, R, RT, and RTp, as illustrated in Figure 13. This shift in connectivity corresponds to the underlying chemoarchitecture: the proportion of input from MGv to each cortical area declines with the intensity of PV immunostaining (Molinari et al., 1995), which is uniformly dense in AI and R, becomes weaker in RT, and tapers off in RTp (Jones et al., 1995; Hackett et al., 1998a; Saleem et al., 2007; Scott et al., 2017). The tapering of MGv input in rostral auditory cortex is balanced by a greater proportion of inputs from the non-lemniscal auditory pathway, and from auditory-related multisensory nuclei outside the MGN.

Quantifying the thalamic inputs to each of these areas addresses several outstanding questions about the connectivity and function of auditory cortex (discussed below), beginning with the extent of auditory cortex itself. As a working definition, 'auditory cortex' consists of those cortical areas receiving significant input from the MGN (i.e. the core, belt, and parabelt), which occupy roughly the caudal two-thirds of the STP and STG (Hackett, 2011). The MGN input to RTp in the present study suggests that the auditory cortex proper extends farther rostrally on the STP, to within about $3 \mathrm{~mm}$ of the temporal pole.

\section{Areas $\mathbf{A l}$ and $\mathbf{R}$}

Both $\mathrm{AI}$ and $\mathrm{R}$ received strong thalamic inputs from the $\mathrm{MGv}$, as has been established in prior studies of macaques (Mesulam and Pandya, 1973; Morel et al., 1993; Molinari et al., 1995; Rauschecker et al., 1997; Hackett et al., 2007) and New World monkeys (Burton and Jones, 1976; Luethke et al., 1989; Morel and Kaas, 1992; de la Mothe et al., 2006b). Despite variability between cases in each area, the overall proportion of MGv input was strikingly similar between AI and R ( $88 \%$ and $85 \%$, respectively), and comparable to other quantitative studies in the literature. As seen in Table 4, the data from AI are skewed by the large number of labeled cells following the DY injection (case 2, 97\% MGv), but the other two cases had 55-60\% MGv label, in accord with prior data from marmosets (60-70\% MGv label in AI and R; (de la Mothe et al., 2006b). Although MGv is taken to be synonymous with the lemniscal (primary) auditory pathway, MGad is also likely to be part of the lemniscal pathway on the basis of its physiological properties and immunostaining for PV (Molinari et al., 1995; Hackett et al., 2007; Bartlett and Wang, 2011). Only a single injection, in AI near the border with R, resulted in significant label in MGad (case OR-1; $60 \% \mathrm{MGv}, 36 \% \mathrm{MGad}$ ), which was comparable to an injection into caudal AI reported by Hackett and colleagues (2007). In the present study no significant MGad connections were evident rostral to the AI/R border (Fig. 13A,B), in accord with prior reports (Molinari et al., 1995; de la Mothe et al., 2006b; 2012b).

Their nearly identical cyto- and chemo-architecture and similarly strong MGv input support the inclusion of AI and R as a unified "core" field. Although both areas receive input from 
the MGv, they do not share the same MGv inputs. As shown in Figure 14, MGv cells projecting to AI were predominantly located in the rostral half of the nucleus, whereas cells projecting to R, RT, or RTp were confined to the caudal half (see Fig. 4 for a dual injection in a single case). This corroborates prior observations that inputs to the caudal auditory cortex typically arise from the rostral MGN, and vice-versa (Mesulam and Pandya, 1973; Burton and Jones, 1976; Jones and Burton, 1976; Morel and Kaas, 1992; Morel et al., 1993; Molinari et al., 1995; Rauschecker et al., 1997; Hackett et al., 1998b; de la Mothe et al., 2006b).Although clusters of cells projecting to caudal and rostral core did overlap (e.g., Fig. 4C), we observed no double-labeled cells in our data (i.e., single cells projecting to both caudal and rostral core). One caveat is that injection sites in the present study were not equated for frequency tuning, so the segregation of labeled cells could, in principle, result from injections into cortical regions with disparate frequency preference. The axis of tonotopy in the primate MGv has not been defined, but some evidence suggests a dorsal/ ventral segregation (Morel et al., 1993), while Jones (2007) has argued for a lateral/medial gradient (inferred from the work of Molinari et al. 1995, in which tonotopy was not measured), but no evidence indicates a rostral/caudal tonotopic axis in MGv. In one prior study that did control for frequency preference across dual injections into AI and R, only a few examples of double-labeled neurons in MGv were noted (Rauschecker et al., 1997). Molinari and colleagues (1995) described counting double-labeled cells, but they showed only non-overlapping clusters in the MGv. Based on the available evidence, the MGv inputs to $\mathrm{AI}$ and $\mathrm{R}$ appear to arise from mostly independent populations within that subdivision.

In accord with their similar structure and connectivity, AI and R share many functional similarities: single neurons in awake AI and $\mathrm{R}$ show similar spontaneous and driven firing rates, low thresholds for tonal stimulation, and sharp frequency tuning (Recanzone et al., 2000; Scott et al., 2011). However, there is a functional gradient of temporal response properties between the caudal and rostral core, such that neurons in $\mathrm{R}$ have longer response latencies and less precise synchronization of their spike timing to the envelope of rapidly modulating stimuli (Scott et al., 2011; Camalier et al., 2012); in the marmoset, this gradient has been shown to extend into RT (Bendor and Wang, 2008). In the marmoset MGv, neurons with non-synchronized responses or a preference for slower modulation frequencies were found caudally (Bartlett and Wang, 2011), i.e., within the region of MGv projecting to R and RT. In the MGv of the cat, both response latency and the prevalence of non-monotonic ratelevel functions increase from rostral to caudal, as does the density of inhibitory (GABAcontaining) neurons (Rouiller et al., 1990). If the same were true in macaques, then stronger inhibition in the caudal MGv could contribute to the greater prevalence of non-monotonic rate-level functions in R relative to AI (Recanzone et al., 2000; Scott et al., 2011). Although the role of cortico-cortical connections cannot be discounted (Rauschecker et al., 1997; Scott et al., 2017), the differences in response timing and sound level tuning between AI and R are likely to be inherited, at least in part, from distinct pools of thalamic inputs (Smith, 2011).

\section{Area RT}

Whether RT should be considered part of the core auditory cortex has been uncertain since the area was identified in macaques (Hackett et al., 1998a). This area had earlier been distinguished as parakoniocortex (area 'paAr') that was more like the granular koniocortex 
of the core than the surrounding belt region (Pandya and Sanides, 1973). By a similar logic, the dense staining for PV, acetylcholinesterase, and myelin that define the core are less distinct in $\mathrm{RT}$ than in $\mathrm{AI}$ and $\mathrm{R}$, but more similar to core than to the surrounding belt (Hackett et al., 1998a). Coupled with connectional evidence that this area did not connect strongly to parabelt, RT was tentatively assigned to the core pending further data on its connections and physiology (Hackett et al., 1998a).

Consistent with its anatomical characteristics that are intermediate between core and belt, the corticocortical and corticothalamic connections of RT are subtly distinct from those of AI and R. Tracer injections into RT identified predominantly local connections to adjacent areas of the STP and moderate direct connections to the parabelt, a pattern similar to that seen after injections into AI and R (Scott et al., 2017). Considered from the perspective of hierarchical connectivity (Rockland and Pandya, 1979; Felleman and Van Essen, 1991; Hegde and Felleman, 2007), connections between AI and R were consistent with a lateral (within-level) projection (Scott et al., 2017; marmoset: de la Mothe 2006a). However, anterograde tracers injected into R identified inputs to RT targeting layer 4 and deep layer 3 that are characteristic of a feed-forward projection (Scott et al., 2017), which contradicts the idea that AI, R and RT comprise a single hierarchical level.

The present data confirm that RT receives thalamic input from MGv in parallel with AI and $\mathrm{R}$, which rules out a strict hierarchical step between them; but RT receives a higher proportion of its thalamic input from MGpd, a distribution qualitatively different from that in AI and R (Figure 13A). One caveat is that some injections into RT were fairly shallow (Figs. $4,6,7)$, which may bias the resulting label toward MGd because calbindin-positive thalamic cells project preferentially to layers 1-2, whereas parvalbumin-positive cells project to layers 3-5 (Hashikawa et al., 1995; Molinari et al., 1995). In addition, the injections in case 9 (rostral RT) and case 10 (RT/RTp border) both resulted in $>60 \%$ of labeled thalamic cells in MGv (Table 4; note that the border case was not included in summary Figure 13A). Taking the variability among injections into account, the thalamic connections of area RT may less starkly different from those of $\mathrm{AI}$ and $\mathrm{R}$ as Figure 13A implies.

Although RT has been identified in New World monkeys, including owl monkeys and marmosets (Morel and Kaas, 1992; de la Mothe et al., 2006a), among Old World monkeys RT was consistently observed in M. mulatta (used here) but not in M. nemestrina ((Hackett et al., 1998a). Species or individual differences may explain why one prior study that placed injections into the rostral STP in M. fuscata (areas that roughly correspond to RT and RTp) found labeled cells in MGpd, MGm, and the suprageniculate nucleus, but not in MGv (Molinari et al. 1995, their Fig. 9).

Published data on the physiology of area RT and the subdivisions of the MGN are scarce in the macaque monkey, but the functional implications of the thalamic inputs to RT can be inferred from recordings in the awake marmoset. Responses to pure-tone stimuli, characteristic of core auditory cortex, are less common in RT (60\%) than in AI and R (79\% and $74 \%$, respectively); in addition, response latencies were longer and synchrony to modulated stimuli was weaker in RT relative to AI (Bendor and Wang, 2008). These characteristics are consistent with a greater proportion of inputs from MGpd, where neurons 
are less frequently tone responsive, and less likely to synchronize their discharges, relative to neurons in MGv (Allon and Yeshurun, 1985; Bartlett and Wang, 2011).

In terms of anatomy, connectivity, and physiology, RT is less clearly 'core-like' than AI and $\mathrm{R}$, and may lie at a level intermediate between core and belt. We have previously suggested that in this regard, area RT is the mirror image of caudomedial belt area CM (Scott et al., 2017, p. 837). Area CM exhibits short-latency physiological responses to narrowband sounds (Camalier et al., 2012) despite having the anatomical characteristics of a belt area, including a small proportion of thalamic inputs from MGv: $₫ 3 \%$ in rhesus macaques (Hackett et al., 2007; Rauschecker et al., 1997), and 5\% in marmosets (de la Mothe et al., 2012b). Physiological responses in RT are arguably less 'core-like' than those in CM, but $\mathrm{RT}$ receives nearly half of its thalamic input from MGv - less than AI and R, but clearly more than CM or the rostral belt area RTL adjacent to RT (15\% for RTL in marmoset; de la Mothe et al., 2012b). This apparent paradox between CM and RT might be explained by their differential inputs from the MGd. The short-latency responses in CM have been suggested to arise from $\mathrm{MGad}$, which provides a stronger input to $\mathrm{CM}$ than to its adjacent belt areas (de la Mothe et al., 2006b; Hackett et al., 2007), but none to any areas rostral to AI in the present study.

\section{Area RTp}

Area RTp on the rostral STP was recently identified as rostral extension of area RT on the basis of PV immunostaining (Saleem et al., 2007; Saleem and Logothetis, 2012). This area had previously been grouped with the laterally adjacent STGr as a region of auditory association cortex, area Ts2 ((Galaburda and Pandya, 1983; Cipolloni and Pandya, 1989), but we recently argued that RTp is a distinct area with different architectonic features and cortico-cortical connections (Scott et al., 2017). Area RTp received about $70 \%$ of its thalamic input from the MGN, including 25\% from MGv, which suggests that RTp should be considered part of the auditory cortex. Less clear is the question of how, or whether, RTp fits into the model of core, belt, and parabelt (discussed in Scott et al., 2017). The STGr received comparatively sparse MGN input, and should still be considered 'auditory related' cortex (Figs 12,13A) that receives less MGN input than the adjacent rostral parabelt area (Kosmal et al. 1997; de la Mothe et al., 2012b).

The core areas AI, R, and RT received around $90 \%$ of their thalamic input from some combination of MGv and MGpd, whereas RTp received only 50\%, with the difference made up by a stronger input from the MGm and other multimodal regions ( $\mathrm{Sg} / \mathrm{Lim}$ and PM; Fig. 13A). This qualitative difference distinguishes RTp from the core. Although these same multimodal thalamic areas do project to the rostral parabelt (RPB, caudal to STGr), the MGv does not (Hackett et al., 1998b; de la Mothe et al., 2012b). The consistent finding of MGv input (19-25\% across retrograde tracer injections) is sufficient to distinguish RTp from the rostral parabelt. The thalamic connectivity of RTp corroborates its chemoarchitecture, in that RTp is visibly distinct from the core and RPB in terms of PV or SMI-32 immunostaining (Scott et al., 2017).

This leaves the possibility is that RTp is a continuation of the rostral belt (RTM and RTL), wrapping around area RT. Structurally, RTp is more similar to medial belt area RTM than to 
lateral belt area RTL, in that PV immunoreactivity is restricted to a thin band in and around layer 4, and SMI-32 immunoreactive neurons are concentrated in the deep layers 5 and 6 (Scott et al., 2017). One study quantified the distribution of thalamic label after an injection into RTL in the marmoset, and found about $85 \%$ of thalamic input to originate in the MGN (15\% MGv, 36\% MGpd, and 34\% MGm; (de la Mothe et al., 2012b). The extent of input from outside the MGN, specifically $\mathrm{Sg} / \mathrm{Lim}$ and PM, appears to be sufficient to distinguish RTp from RTL, but more data on the connections of the rostral belt (particularly the medial belt) will be necessary to clarify this distinction. Given the present data, we maintain that RTp does not clearly fall within the core/belt/parabelt model, but appears to represent a point of convergence between multiple cortical and subcortical auditory pathways.

\section{Multimodal interactions and auditory cognition}

Injections into RTp consistently identified connections to the $\mathrm{Sg} / \mathrm{Lim}$ nucleus and the medial pulvinar, to a greater extent than was observed after injections into core areas. Whereas the MGN functions as a conduit between midbrain and cortex, most pulvinar projections are to and from the cortex, with the PM positioned as a central hub connecting a vast area of association cortex (Burton and Jones, 1976; Jones, 2007; Cappe et al., 2009; Bridge et al., 2016). The PM provides the primary thalamocortical projection to the STG across primate species (Burton and Jones, 1976), with its lateral and medial subdivisions engaged in partially overlapping networks. The PMm, where labeled cells were concentrated in our injections, is known to connect with the cingulate cortex, STS, and STG (with a bias toward their rostral portions), as well as the temporal pole, amygdala, and several regions of the PFC (ventrolateral, orbital, and medial; (Jones and Burton, 1976; Romanski et al., 1997; Gutierrez et al., 2000). The PMm and the Sg/Lim may both receive subcortical input from the intermediate and deep layers of the superior colliculus (Benevento and Fallon, 1975; Benevento and Standage, 1983; Jones, 2007). Neurons in the pulvinar respond to both sound and movement during an auditory-guided behavioral task, but not during passive listening (Yirmiya and Hocherman, 1987), suggesting a role in sensory-motor interactions that is consistent with the connectivity of PM (Cappe et al., 2009).

Multisensory and task-dependent activity that has been observed in RTp and nearby areas may be facilitated by thalamic connections to PM and to non-lemniscal auditory thalamus (MGm and MGpd), which receives inputs from outside the auditory system and provides neuromodulatory inputs to cortex (Edeline, 2012; Lee, 2015). These connections, along with the widespread connections of PM with PFC and association cortex, may contribute to modulations of activity in RTp and surrounding areas that have been associated with performance of auditory short-term memory tasks (Ng et al., 2014; Scott et al., 2014). The possible role of these areas in short-term memory is supported by the presence of stronger stimulus-specific adaptation in non-lemniscal auditory thalamus (Nieto-Diego and Malmierca, 2016), which provides a greater proportion of thalamic input to the rostral auditory areas. Audiovisual interactions have been studied in a region of the rostral STP that is sensitive to monkey vocalizations, and is likely coextensive with area RTp (Perrodin et al., $2011 ; 2014)$. Although $41 \%$ of neurons responsive to monkey vocalizations were influenced by visual stimuli, these effects were not specific to congruent face/voice pairings, in contrast to the association cortex of the superior temporal sulcus (Perrodin et al., 2014). This 
suggests that whereas STS receives highly refined visual input from inferotemporal visual cortex (Grimaldi et al., 2016), RTp may be influenced by less specific visual inputs arriving via PM or Sg/Lim (Cappe et al., 2009), in addition to indirect input from the STS (via the STGr; Scott et al., 2017).

A few RTp injections resulted in sparse label within the mediodorsal thalamus, specifically its posterior subdivision MDpc. The few auditory responses that have been recorded within MD thalamus were confined to this region (Tanibuchi and Goldman-Rakic, 2003). The MD thalamus plays a role in working memory, including an auditory memory circuit in which MD receives input from the rostral STG, but these inputs are predominantly within more anterior MD subdivisions (Munoz et al., 2009). The MDpc projects to medial prefrontal areas 10 and 14 (Bachevalier et al., 1997), the same areas also interconnected with rostral auditory cortex area RTp (our data, unpublished observations; see also Romanski et al., 1997). These anatomical connections suggest that information from MDpc may reach the medial prefrontal areas directly, or indirectly via the higher auditory cortex. Neurons in MDpc and PM are selectively compromised in the brains of schizophrenics (Popken et al., 2000; Danos et al., 2003), who also exhibit hypoactivity in PFC and abnormalities of working memory and auditory processing (Javitt and Sweet, 2015). This is consistent with the thalamocortical circuits of the rostral STG and STP playing a key role in auditory and social cognition (Perrodin et al., 2015).

\section{Corticothalamic projections}

The ascending auditory pathway from the periphery to the cortex is complemented by an extensive descending system that may exert a profound influence on sensory processing (Winer, 2006). Consistent with prior studies in cats (Winer et al., 2001) and primates (FitzPatrick and Imig, 1978; Pandya et al., 1994; Rouiller and Durif, 2004; de la Mothe et al., 2006b), thalamocortical projections were reciprocated by descending corticothalamic projections. Like the ascending projections, these descending projections were increasingly divergent in more rostral cortical areas such that $\mathrm{AI}$ and $\mathrm{R}$ projected most strongly to $\mathrm{MGv}$, area RT projected to MGv and MGpd, and area RTp projected to MGv and MGpd as well as to MGm and PM. Corticothalamic projections allow a given cortical area to exert active, specific, and adaptive control over its thalamic input by direct feedback on excitatory projection neurons or inhibitory interneurons, or by neuromodulatory influences (Winer, 2006; Edeline, 2012; Lee, 2015).

Reciprocal connections offer a path by which cortical areas can directly modulate their inputs, but not all corticothalamic connections were reciprocal: in several cases fields of labeled terminals without filled cells were evident, particularly in MGpd (e.g., Figs. 5, 7, 10). These non-reciprocal connections have been hypothesized to play a role in an emerging model of thalamocortical function in which information is transferred from lower to higher cortical stages not only by direct cortico-cortical connections, but also by indirect corticothalamo-cortical loops (Lee and Sherman, 2011; Lee, 2015). For example, information may flow between R and RT by direct feedforward inputs, as well as by the projection from R to MGpd, which in turn projects to RT; the same motif may repeat between RT and RTp. These pathways are consistent with the emerging idea that thalamus is not a simple relay to the 
initial stage of cortical processing, but that recurrent trans-thalamic loops provide a basis for the thalamus to contribute to the processing of information throughout the cortical hierarchy (Sherman, 2016).

\section{Other Acknowledgements}

We thank P. Leccese, M. Mullarkey, D. Yu, A. Cummins, H. Vinal, A. Kloth, R. Reoli, C. Blackwell, A. Doyle, and M. Muñoz-Lopez for technical assistance and advice. This work was supported by the Division of Intramural Research Programs, National Institute of Mental Health, National Institutes of Health. Correspondence may be addressed to brianScott@mail.nih.gov or bhScott@outlook.com.

Supported by: Division of Intramural Research Programs grant ZIA MH001101, National Institute of Mental Health, NIH.

\section{Literature Cited}

Allon N, Yeshurun Y. 1985 Functional organization of the medial geniculate body's subdivisions of the awake squirrel monkey. Brain research 360(1-2):75-82. [PubMed: 4075184]

Bachevalier J, Meunier M, Lu MX, Ungerleider LG. 1997 Thalamic and temporal cortex input to medial prefrontal cortex in rhesus monkeys. Experimental brain research 115(3):430-444. [PubMed: 9262198]

Bartlett EL. 2013 The organization and physiology of the auditory thalamus and its role in processing acoustic features important for speech perception. Brain Lang 126(1):29-48. [PubMed: 23725661]

Bartlett EL, Wang X. 2011 Correlation of neural response properties with auditory thalamus subdivisions in the awake marmoset. Journal of neurophysiology 105(6):2647-2667. [PubMed: 21411564]

Bendor D, Wang X. 2008 Neural response properties of primary, rostral, and rostrotemporal core fields in the auditory cortex of marmoset monkeys. Journal of neurophysiology 100(2):888-906. [PubMed: 18525020]

Benevento LA, Fallon JH. 1975 The ascending projections of the superior colliculus in the rhesus monkey (Macaca mulatta). The Journal of comparative neurology 160(3):339-361. [PubMed: 1112928]

Benevento LA, Standage GP. 1983 The organization of projections of the retinorecipient and nonretinorecipient nuclei of the pretectal complex and layers of the superior colliculus to the lateral pulvinar and medial pulvinar in the macaque monkey. The Journal of comparative neurology 217(3):307-336. [PubMed: 6886056]

Bridge H, Leopold DA, Bourne JA. 2016 Adaptive Pulvinar Circuitry Supports Visual Cognition. Trends in cognitive sciences 20(2):146-157. [PubMed: 26553222]

Burton H, Jones EG. 1976 The posterior thalamic region and its cortical projection in New World and Old World monkeys. The Journal of comparative neurology 168(2):249-301. [PubMed: 821975]

Camalier CR, D'Angelo WR, Sterbing-D'Angelo SJ, de la Mothe LA, Hackett TA. 2012 Neural latencies across auditory cortex of macaque support a dorsal stream supramodal timing advantage in primates. Proceedings of the National Academy of Sciences of the United States of America 109(44):18168-18173. [PubMed: 23074251]

Cappe C, Morel A, Barone P, Rouiller EM. 2009 The thalamocortical projection systems in primate: an anatomical support for multisensory and sensorimotor interplay. Cerebral cortex 19(9):2025-2037. [PubMed: 19150924]

Celio MR. 1986 Parvalbumin in most gamma-aminobutyric acid-containing neurons of the rat cerebral cortex. Science 231(4741):995-997. [PubMed: 3945815]

Cipolloni PB, Pandya DN. 1989 Connectional analysis of the ipsilateral and contralateral afferent neurons of the superior temporal region in the rhesus monkey. The Journal of comparative neurology 281(4):567-585. [PubMed: 2708582] 
Danos P, Baumann B, Kramer A, Bernstein HG, Stauch R, Krell D, Falkai P, Bogerts B. 2003 Volumes of association thalamic nuclei in schizophrenia: a postmortem study. Schizophr Res 60(2-3):141155. [PubMed: 12591578]

de la Mothe LA, Blumell S, Kajikawa Y, Hackett TA. 2006a Cortical connections of the auditory cortex in marmoset monkeys: core and medial belt regions. The Journal of comparative neurology 496(1):27-71. [PubMed: 16528722]

de la Mothe LA, Blumell S, Kajikawa Y, Hackett TA. 2006b Thalamic connections of the auditory cortex in marmoset monkeys: core and medial belt regions. The Journal of comparative neurology 496(1):72-96. [PubMed: 16528728]

de la Mothe LA, Blumell S, Kajikawa Y, Hackett TA. 2012a Cortical connections of auditory cortex in marmoset monkeys: lateral belt and parabelt regions. Anatomical record 295(5):800-821.

de la Mothe LA, Blumell S, Kajikawa Y, Hackett TA. 2012b Thalamic connections of auditory cortex in marmoset monkeys: lateral belt and parabelt regions. Anatomical record 295(5):822-836.

Edeline JM. 2012 Beyond traditional approaches to understanding the functional role of neuromodulators in sensory cortices. Front Behav Neurosci 6:45. [PubMed: 22866031]

Edeline JM, Manunta Y, Nodal FR, Bajo VM. 1999 Do auditory responses recorded from awake animals reflect the anatomical parcellation of the auditory thalamus? Hearing research 131(1-2): 135-152. [PubMed: 10355611]

Felleman DJ, Van Essen DC. 1991 Distributed hierarchical processing in the primate cerebral cortex. Cerebral cortex 1(1):1-47. [PubMed: 1822724]

FitzPatrick KA, Imig TJ. 1978 Projections of auditory cortex upon the thalamus and midbrain in the owl monkey. The Journal of comparative neurology 177(4):573-555. [PubMed: 415070]

Fukushima M, Saunders RC, Leopold DA, Mishkin M, Averbeck BB. 2012 Spontaneous high-gamma band activity reflects functional organization of auditory cortex in the awake macaque. Neuron 74(5):899-910. [PubMed: 22681693]

Fukushima M, Saunders RC, Leopold DA, Mishkin M, Averbeck BB. 2014 Differential coding of conspecific vocalizations in the ventral auditory cortical stream. The Journal of neuroscience : the official journal of the Society for Neuroscience 34(13):4665-4676. [PubMed: 24672012]

Galaburda AM, Pandya DN. 1983 The intrinsic architectonic and connectional organization of the superior temporal region of the rhesus monkey. The Journal of comparative neurology 221(2):169_ 184. [PubMed: 6655080]

Grimaldi P, Saleem KS, Tsao D. 2016 Anatomical Connections of the Functionally Defined "Face Patches" in the Macaque Monkey. Neuron 90(6):1325-1342. [PubMed: 27263973]

Gutierrez C, Cola MG, Seltzer B, Cusick C. 2000 Neurochemical and connectional organization of the dorsal pulvinar complex in monkeys. The Journal of comparative neurology 419(1):61-86. [PubMed: 10717640]

Hackett TA. 2011 Information flow in the auditory cortical network. Hearing research 271(1-2):133146. [PubMed: 20116421]

Hackett TA, De La Mothe LA, Ulbert I, Karmos G, Smiley J, Schroeder CE. 2007 Multisensory convergence in auditory cortex, II. Thalamocortical connections of the caudal superior temporal plane. The Journal of comparative neurology 502(6):924-952. [PubMed: 17444488]

Hackett TA, Stepniewska I, Kaas JH. 1998a Subdivisions of auditory cortex and ipsilateral cortical connections of the parabelt auditory cortex in macaque monkeys. The Journal of comparative neurology 394(4):475-495. [PubMed: 9590556]

Hackett TA, Stepniewska I, Kaas JH. 1998b Thalamocortical connections of the parabelt auditory cortex in macaque monkeys. The Journal of comparative neurology 400(2):271-286. [PubMed: 9766404]

Hashikawa T, Molinari M, Rausell E, Jones EG. 1995 Patchy and laminar terminations of medial geniculate axons in monkey auditory cortex. The Journal of comparative neurology 362(2):195208. [PubMed: 8576433]

Hashikawa T, Rausell E, Molinari M, Jones EG. 1991 Parvalbumin- and calbindin-containing neurons in the monkey medial geniculate complex: differential distribution and cortical layer specific projections. Brain research 544(2):335-341. [PubMed: 2039948] 
Hegde J, Felleman DJ. 2007 Reappraising the functional implications of the primate visual anatomical hierarchy. Neuroscientist 13(5):416-421. [PubMed: 17901251]

Hendry SH, Jones EG, Emson PC, Lawson DE, Heizmann CW, Streit P. 1989 Two classes of cortical GABA neurons defined by differential calcium binding protein immunoreactivities. Experimental brain research 76(2):467-472. [PubMed: 2767197]

Javitt DC, Sweet RA. 2015 Auditory dysfunction in schizophrenia: integrating clinical and basic features. Nat Rev Neurosci 16(9):535-550. [PubMed: 26289573]

Jones EG. 2007 The thalamus. Cambridge ; New York: Cambridge University Press.

Jones EG, Burton H. 1976 Areal differences in the laminar distribution of thalamic afferents in cortical fields of the insular, parietal and temporal regions of primates. The Journal of comparative neurology 168(2):197-247. [PubMed: 821974]

Jones EG, Dell'Anna ME, Molinari M, Rausell E, Hashikawa T. 1995 Subdivisions of macaque monkey auditory cortex revealed by calcium-binding protein immunoreactivity. The Journal of comparative neurology 362(2):153-170. [PubMed: 8576431]

Kaas JH, Hackett TA. 1998 Subdivisions of auditory cortex and levels of processing in primates. Audiology \& neuro-otology 3(2-3):73-85. [PubMed: 9575378]

Kikuchi Y, Horwitz B, Mishkin M. 2010 Hierarchical auditory processing directed rostrally along the monkey's supratemporal plane. The Journal of neuroscience : the official journal of the Society for Neuroscience 30(39):13021-13030. [PubMed: 20881120]

Lee CC. 2015 Exploring functions for the non-lemniscal auditory thalamus. Front Neural Circuits 9:69. [PubMed: 26582978]

Lee CC, Sherman SM. 2011 On the classification of pathways in the auditory midbrain, thalamus, and cortex. Hearing research 276(1-2):79-87. [PubMed: 21184817]

Luethke LE, Krubitzer LA, Kaas JH. 1989 Connections of primary auditory cortex in the New World monkey, Saguinus. The Journal of comparative neurology 285(4):487-513. [PubMed: 2474584]

Merzenich MM, Brugge JF. 1973 Representation of the cochlear partition of the superior temporal plane of the macaque monkey. Brain research 50(2):275-296. [PubMed: 4196192]

Mesulam MM, Pandya DN. 1973 The projections of the medial geniculate complex within the sylvian fissure of the rhesus monkey. Brain research 60(2):315-333. [PubMed: 4202852]

Molinari M, Dell'Anna ME, Rausell E, Leggio MG, Hashikawa T, Jones EG. 1995 Auditory thalamocortical pathways defined in monkeys by calcium-binding protein immunoreactivity. The Journal of comparative neurology 362(2):171-194. [PubMed: 8576432]

Morel A, Garraghty PE, Kaas JH. 1993 Tonotopic organization, architectonic fields, and connections of auditory cortex in macaque monkeys. The Journal of comparative neurology 335(3):437-459. [PubMed: 7693772]

Morel A, Kaas JH. 1992 Subdivisions and connections of auditory cortex in owl monkeys. The Journal of comparative neurology 318(1):27-63. [PubMed: 1583155]

Munoz M, Mishkin M, Saunders RC. 2009 Resection of the medial temporal lobe disconnects the rostral superior temporal gyrus from some of its projection targets in the frontal lobe and thalamus. Cerebral cortex 19(9):2114-2130. [PubMed: 19150921]

Ng CW, Plakke B, Poremba A. 2013 Neural correlates of auditory recognition memory in the primate dorsal temporal pole. Journal of neurophysiology.

Ng CW, Plakke B, Poremba A. 2014 Neural correlates of auditory recognition memory in the primate dorsal temporal pole. Journal of neurophysiology 111(3):455-469. [PubMed: 24198324]

Nieto-Diego J, Malmierca MS. 2016 Topographic Distribution of Stimulus-Specific Adaptation across Auditory Cortical Fields in the Anesthetized Rat. PLoS biology 14(3):e1002397. [PubMed: 26950883]

Pandya DN, Rosene DL, Doolittle AM. 1994 Corticothalamic connections of auditory-related areas of the temporal lobe in the rhesus monkey. The Journal of comparative neurology 345(3):447-471. [PubMed: 7929912]

Pandya DN, Sanides F. 1973 Architectonic parcellation of the temporal operculum in rhesus monkey and its projection pattern. Zeitschrift fur Anatomie und Entwicklungsgeschichte 139(2):127-161. [PubMed: 4197942] 
Paxinos G, Huang XF, Toga AW. 2000 The rhesus monkey brain in stereotaxic coordinates. San Diego, CA: Academic Press. 163 p. p.

Perrodin C, Kayser C, Abel TJ, Logothetis NK, Petkov CI. 2015 Who is That? Brain Networks and Mechanisms for Identifying Individuals. Trends in cognitive sciences 19(12):783-796. [PubMed: 26454482]

Perrodin C, Kayser C, Logothetis NK, Petkov CI. 2011 Voice cells in the primate temporal lobe. Current biology : CB 21(16):1408-1415. [PubMed: 21835625]

Perrodin C, Kayser C, Logothetis NK, Petkov CI. 2014 Auditory and visual modulation of temporal lobe neurons in voice-sensitive and association cortices. The Journal of neuroscience : the official journal of the Society for Neuroscience 34(7):2524-2537. [PubMed: 24523543]

Petkov CI, Kayser C, Augath M, Logothetis NK. 2006 Functional imaging reveals numerous fields in the monkey auditory cortex. PLoS biology 4(7):e215. [PubMed: 16774452]

Petkov CI, Kayser C, Steudel T, Whittingstall K, Augath M, Logothetis NK. 2008 A voice region in the monkey brain. Nature neuroscience 11(3):367-374. [PubMed: 18264095]

Popken GJ, Bunney WE, Jr., Potkin SG, Jones EG. 2000 Subnucleus-specific loss of neurons in medial thalamus of schizophrenics. Proceedings of the National Academy of Sciences of the United States of America 97(16):9276-9280. [PubMed: 10908653]

Poremba A, Malloy M, Saunders RC, Carson RE, Herscovitch P, Mishkin M. 2004 Species-specific calls evoke asymmetric activity in the monkey's temporal poles. Nature 427(6973):448-451. [PubMed: 14749833]

Poremba A, Saunders RC, Crane AM, Cook M, Sokoloff L, Mishkin M. 2003 Functional mapping of the primate auditory system. Science 299(5606):568-572. [PubMed: 12543977]

Preuss A, Muller-Preuss P. 1990 Processing of amplitude modulated sounds in the medial geniculate body of squirrel monkeys. Experimental brain research 79(1):207-211. [PubMed: 2311698]

Rauschecker JP. 1998 Parallel processing in the auditory cortex of primates. Audiology \& neurootology 3(2-3):86-103. [PubMed: 9575379]

Rauschecker JP, Scott SK. 2009 Maps and streams in the auditory cortex: nonhuman primates illuminate human speech processing. Nature neuroscience 12(6):718-724. [PubMed: 19471271]

Rauschecker JP, Tian B, Pons T, Mishkin M. 1997 Serial and parallel processing in rhesus monkey auditory cortex. The Journal of comparative neurology 382(1):89-103. [PubMed: 9136813]

Recanzone GH, Guard DC, Phan ML. 2000 Frequency and intensity response properties of single neurons in the auditory cortex of the behaving macaque monkey. Journal of neurophysiology 83(4):2315-2331. [PubMed: 10758136]

Rockland KS, Pandya DN. 1979 Laminar origins and terminations of cortical connections of the occipital lobe in the rhesus monkey. Brain research 179(1):3-20. [PubMed: 116716]

Romanski LM, Giguere M, Bates JF, Goldman-Rakic PS. 1997 Topographic organization of medial pulvinar connections with the prefrontal cortex in the rhesus monkey. The Journal of comparative neurology 379(3):313-332. [PubMed: 9067827]

Romanski LM, Tian B, Fritz J, Mishkin M, Goldman-Rakic PS, Rauschecker JP. 1999 Dual streams of auditory afferents target multiple domains in the primate prefrontal cortex. Nature neuroscience 2(12):1131-1136. [PubMed: 10570492]

Rosene DL, Roy NJ, Davis BJ. 1986 A cryoprotection method that facilitates cutting frozen sections of whole monkey brains for histological and histochemical processing without freezing artifact. The journal of histochemistry and cytochemistry : official journal of the Histochemistry Society 34(10):1301-1315. [PubMed: 3745909]

Rouiller EM, Capt M, Hornung JP, Streit P. 1990 Correlation between regional changes in the distributions of GABA-containing neurons and unit response properties in the medial geniculate body of the cat. Hearing research 49(1-3):249-258. [PubMed: 2292499]

Rouiller EM, Durif C. 2004 The dual pattern of corticothalamic projection of the primary auditory cortex in macaque monkey. Neurosci Lett 358(1):49-52. [PubMed: 15016432]

Saleem KS, Logothetis NK. 2012 A combined MRI and histology atlas of the rhesus monkey brain in stereotaxic coordinates 2nd edition with Horizontal, Coronal and Sagittal series. San Diego, CA: Elsevier/Academic press. 
Saleem KS, Price JL, Hashikawa T. 2007 Cytoarchitectonic and chemoarchitectonic subdivisions of the perirhinal and parahippocampal cortices in macaque monkeys. The Journal of comparative neurology 500(6):973-1006. [PubMed: 17183540]

Scott BH, Leccese PA, Saleem KS, Kikuchi Y, Mullarkey MP, Fukushima M, Mishkin M, Saunders RC. 2017 Intrinsic Connections of the Core Auditory Cortical Regions and Rostral Supratemporal Plane in the Macaque Monkey. Cerebral cortex 27(1):809-840. [PubMed: 26620266]

Scott BH, Malone BJ, Semple MN. 2011 Transformation of temporal processing across auditory cortex of awake macaques. Journal of neurophysiology 105(2):712-730. [PubMed: 21106896]

Scott BH, Mishkin M, Yin P. 2014 Neural correlates of auditory short-term memory in rostral superior temporal cortex. Current biology : CB 24(23):2767-2775. [PubMed: 25456448]

Sherman SM. 2016 Thalamus plays a central role in ongoing cortical functioning. Nature neuroscience 19(4):533-541. [PubMed: 27021938]

Smiley JF, Hackett TA, Ulbert I, Karmas G, Lakatos P, Javitt DC, Schroeder CE. 2007 Multisensory convergence in auditory cortex, I. Cortical connections of the caudal superior temporal plane in macaque monkeys. The Journal of comparative neurology 502(6):894-923. [PubMed: 17447261]

Smith EH. 2011 Temporal processing in the auditory core: transformation or segregation? Journal of neurophysiology 106(6):2791-2793. [PubMed: 21795614]

Tanibuchi I, Goldman-Rakic PS. 2003 Dissociation of spatial-, object-, and sound-coding neurons in the mediodorsal nucleus of the primate thalamus. Journal of neurophysiology 89(2):1067-1077. [PubMed: 12574481]

Winer JA. 2006 Decoding the auditory corticofugal systems. Hearing research 212(1-2):1-8. [PubMed: 16555378]

Winer JA, Diehl JJ, Larue DT. 2001 Projections of auditory cortex to the medial geniculate body of the cat. The Journal of comparative neurology 430(1):27-55. [PubMed: 11135244]

Yirmiya R, Hocherman S. 1987 Auditory- and movement-related neural activity interact in the pulvinar of the behaving rhesus monkey. Brain research 402(1):93-102. [PubMed: 3828792] 
By injecting anatomical tracers along the supratemporal plane of macaque monkeys, the authors quantify how the distribution of thalamic inputs shifts between caudal and rostral areas of the auditory cortex. Input from the primary (lemniscal) auditory pathway extends nearly to the temporal pole, underscoring the complexity of the auditory ventral stream in primates. 
Tracer injection sites

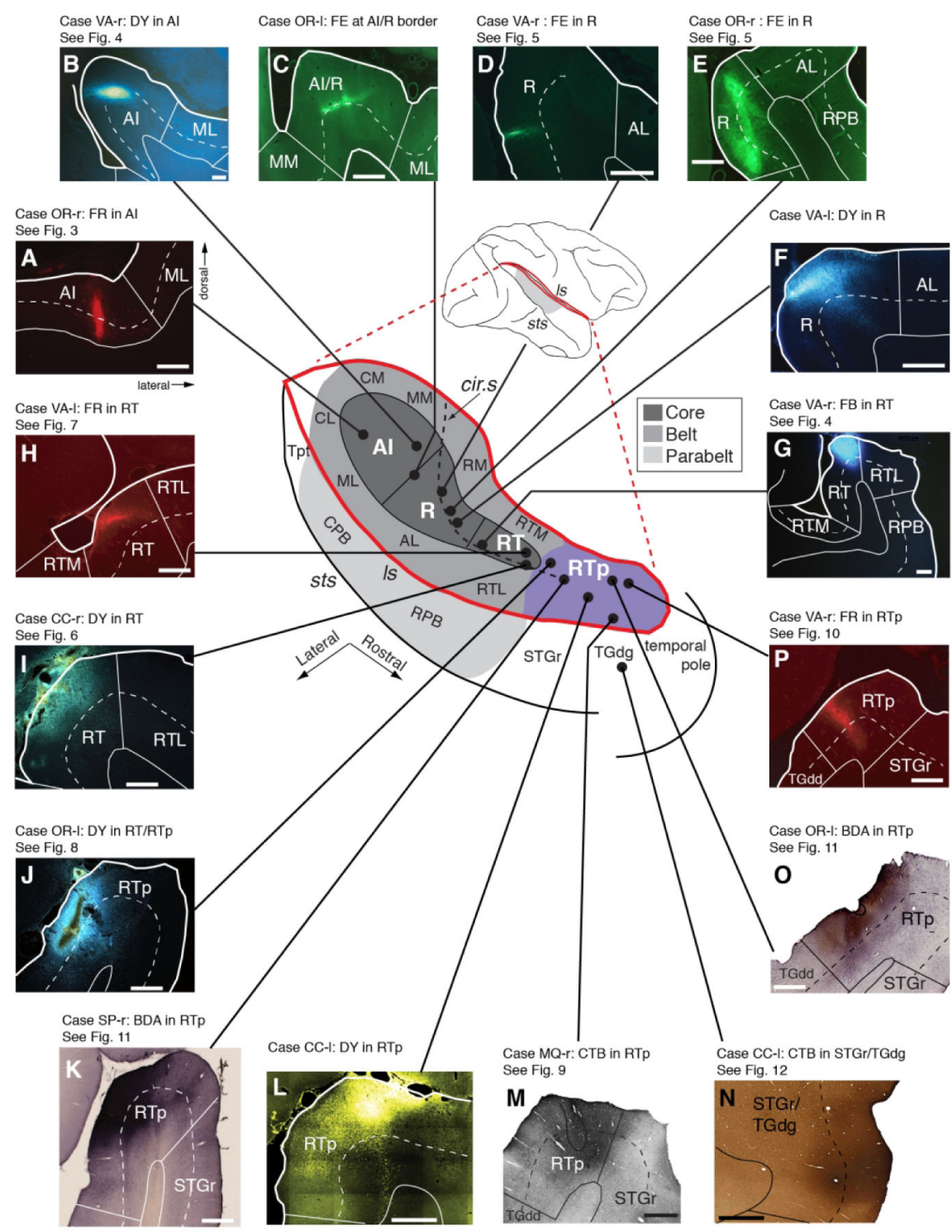

Figure 1.

Subdivisions of the auditory cortex in the supratemporal plane (STP) and the superior temporal gyrus (STG), and the sites of the tracer injections in STP. The lateral sulcus (ls), outlined in red on a lateral view of the macaque right hemisphere (inset), is opened to show three core areas (dark gray), the surrounding belt areas (medium gray), and area RTp (purple) on the surface of the STP, illustrated schematically at the center. The rostrocaudal extent of the parabelt areas (light gray) on the STG is also illustrated in the schematic diagram and on the lateral view of the brain. The curved dashed line within the diagram indicates the approximate location of the lip of the circular sulcus. Black dots mark tracer injection sites along the caudorostral extent of the STP. A photomicrograph of each injection site from caudal to rostral is arrayed clockwise from A to $\mathrm{G}$, and counterclockwise from $\mathrm{H}$ to P. The bold outline indicates the pial surface (in fluorescence photomicrographs only), a 
solid thin line marks the border between the gray and white matter, and a dashed thin line indicates layer 4. The black outline in $\mathrm{M}$ indicates needle damage visible in an adjacent thionine-stained section (not shown). The text above each photomicrograph indicates: 1) Case identifier, composed of a two-letter subject code followed by 'l' or ' $r$ ' to indicate left or right hemisphere, 2) the tracer and target region, and 3) reference to the corresponding data figure, if applicable. In all figures, images from the left hemisphere are flipped to a righthemisphere orientation for consistency. See Table of Abbreviations for the different auditory areas and sulcus designations. Scale bars $=1 \mathrm{~mm}$. 

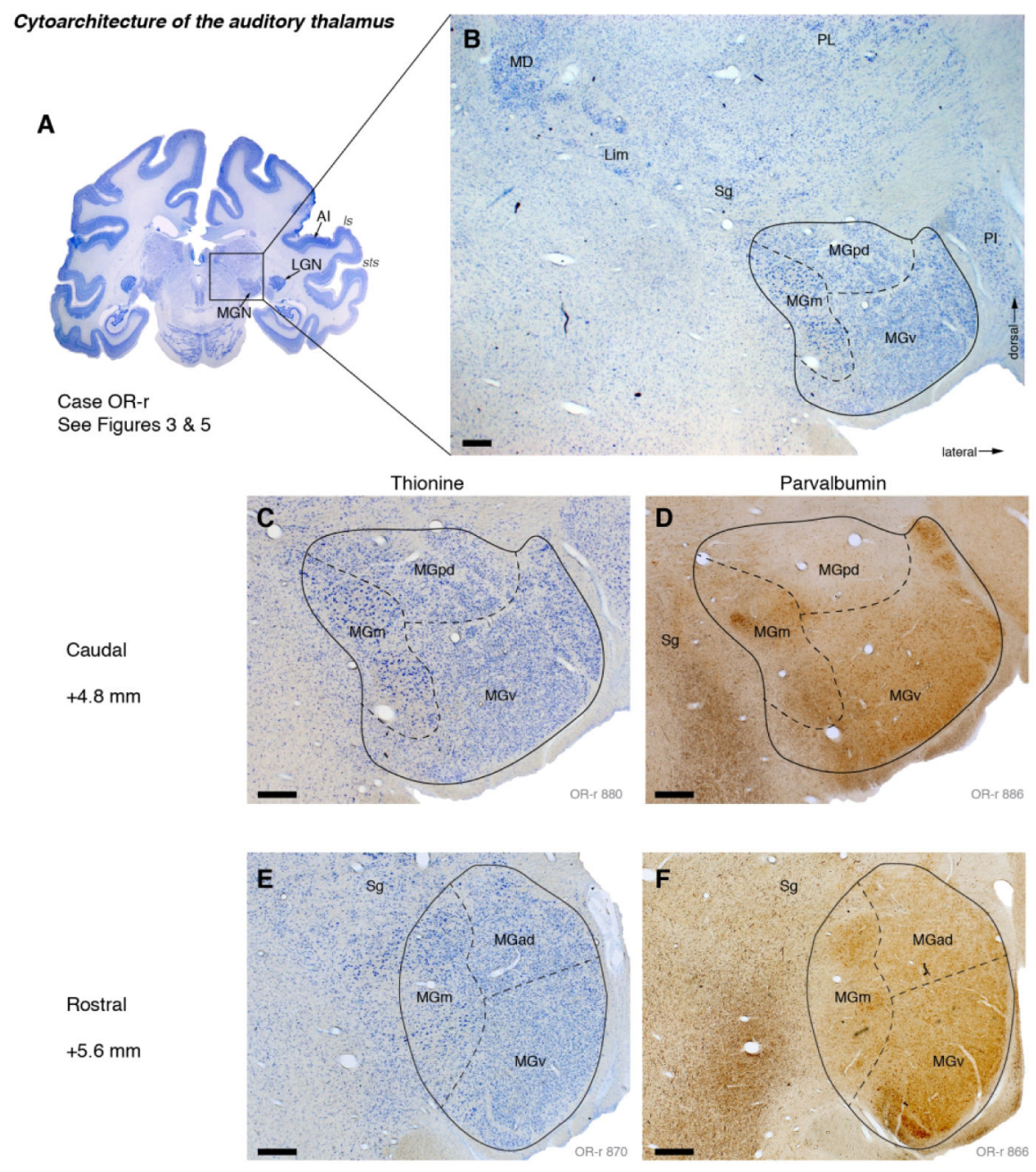

Figure 2.

Cyto- and chemo-architectonic subdivisions of the auditory thalamus in case OR. (A) A thionine-stained coronal section at the level of the MGN. The parietal operculum of the right hemisphere was aspirated to reveal the STP for tracer injection (see Materials and Methods). (B) Higher magnification view of the thalamus, including the ventral, posterodorsal, and magnocellular/medial divisions of the MGN (v, pd, and $\mathrm{m}$, respectively). The suprageniculate ( $\mathrm{Sg}$ ) and limitans ( $\mathrm{Lim}$ ) nuclei extend dorsomedially from the MGN toward the mediodorsal nucleus (MD) of the thalamus. The MGN is bounded laterally by the inferior pulvinar (PI), which is interposed between the MGN and lateral geniculate nucleus (LGN) at this level. (C, D) Higher magnification views of the MGN stained for thionine (same section as B) and a nearby section stained for parvalbumin (PV). (E, F) Like C and D, but farther rostral within the MGN. These sections correspond to Figures 3C-D, and 5B-C. Scale bars $=0.5 \mathrm{~mm}$. 


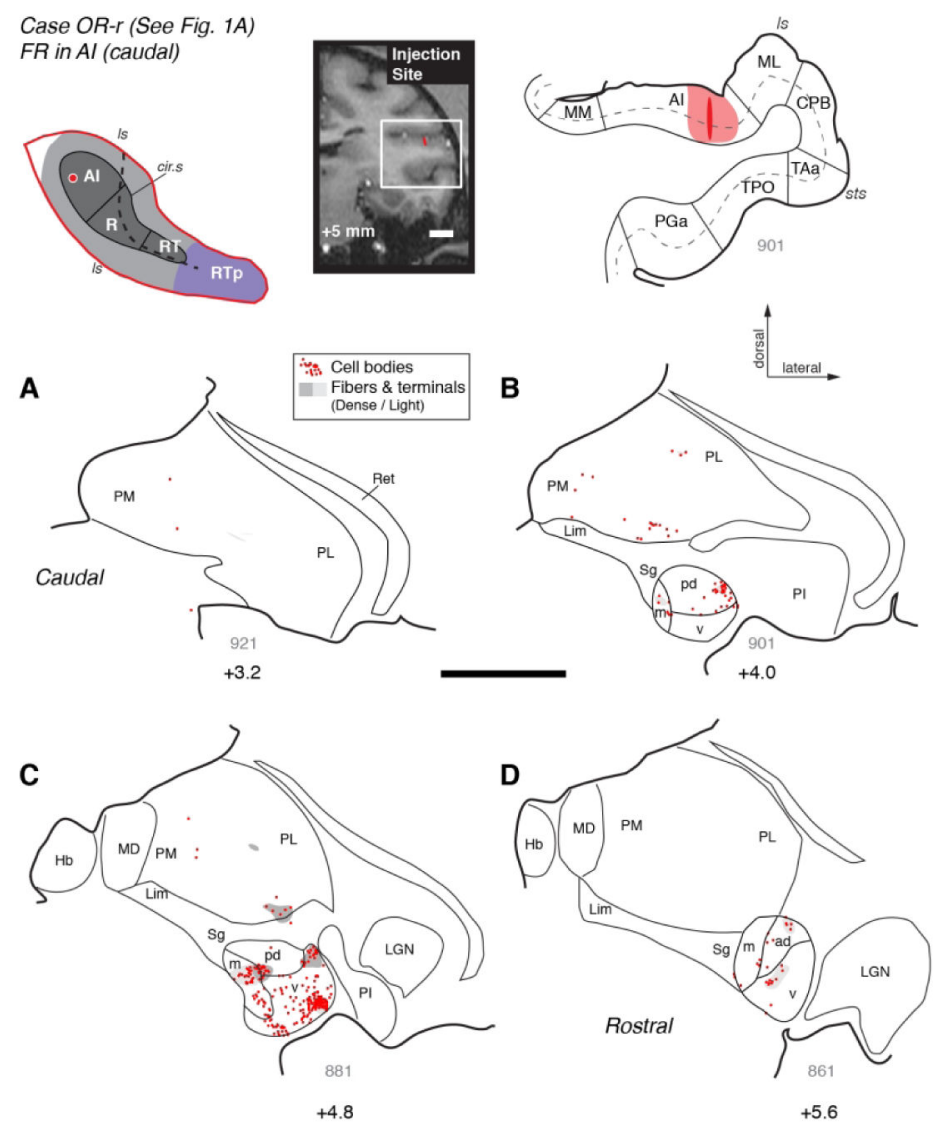

Figure 3.

Thalamic connections of primary auditory area AI in case OR-r. Distribution of retrogradely labeled cells and anterogradely labeled terminals in the MGN and pulvinar after a bidirectional tracer (FR) injection into caudolateral AI (see Fig. 1A). The schematic diagram at the top left indicates the location of the injection site on the STP (as in Fig. 1). The injection site is also indicated on a coronal MRI slice from the same animal (top center), and a corresponding histological section of the superior temporal lobe (top right), outlined by the white rectangle on the MRI. The dark area in the plotted section indicates the needle track, and the surrounding shading indicates the halo around the injection site where background staining was high. The thick line marks the pial surface, thin lines indicate the gray/white matter boundary and borders between cortical areas, and the dashed line indicates cortical layer 4. (A-D) Distribution of label in the MGN and other thalamic nuclei. Only the portion of the coronal section containing the thalamus is depicted, from the medial pulvinar (PM) and habenula $(\mathrm{Hb})$ medially, to the reticular nucleus (Ret) laterally. Sections are arrayed from caudal (A) to rostral (D). Each dot represents one retrogradely labeled cell, and gray shading marks the fields of anterogradely labeled axonal fibers and synaptic terminals (dark and light gray indicate dense and light label, respectively). Thick lines trace the floor of the lateral ventricle and the ventral surface of the brain that delimit the dorsal and ventral extent of the thalamus, respectively. Thin lines mark the borders of thalamic nuclei and the subdivisions of the MGN. The approximate caudorostral position relative to the interaural axis is indicated beneath each section, along with the section number in gray (thickness $=40$ 
$\mu \mathrm{m})$. This AI injection labeled neurons within the MGN (>90\% of thalamic label), primarily within the MGv, with smaller populations in the MGm and MGpd/ad. Anterograde label was co-localized with a cluster of labeled cells in ventral MGv (C), and was also found in MGm, MGpd, and ventral pulvinar (C). See Table 4 for cell counts in each nucleus and subdivision. See Table of Abbreviations for area, nucleus, and sulcus designations. Scale bars $=5 \mathrm{~mm}$. 
Case VA-r (see Figs. 1B, 1G) DY in Al (rostral) FB in RT (caudal)

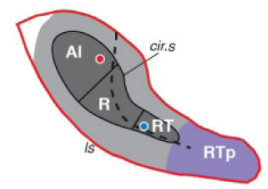

A

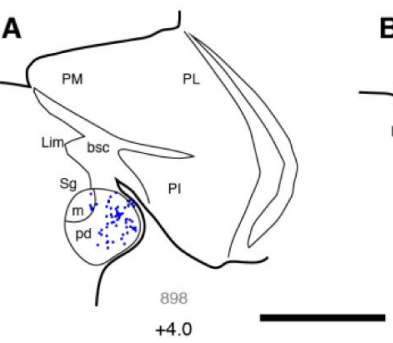

$+4.0$

D

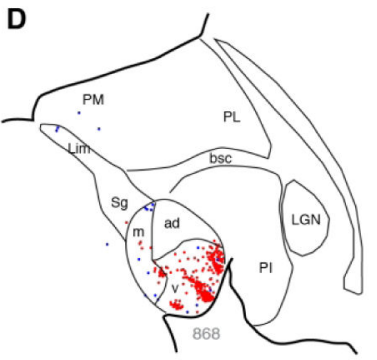

$+5.2$

E

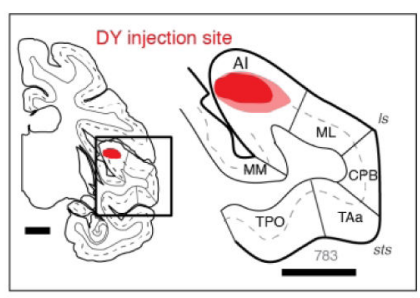

B B PM
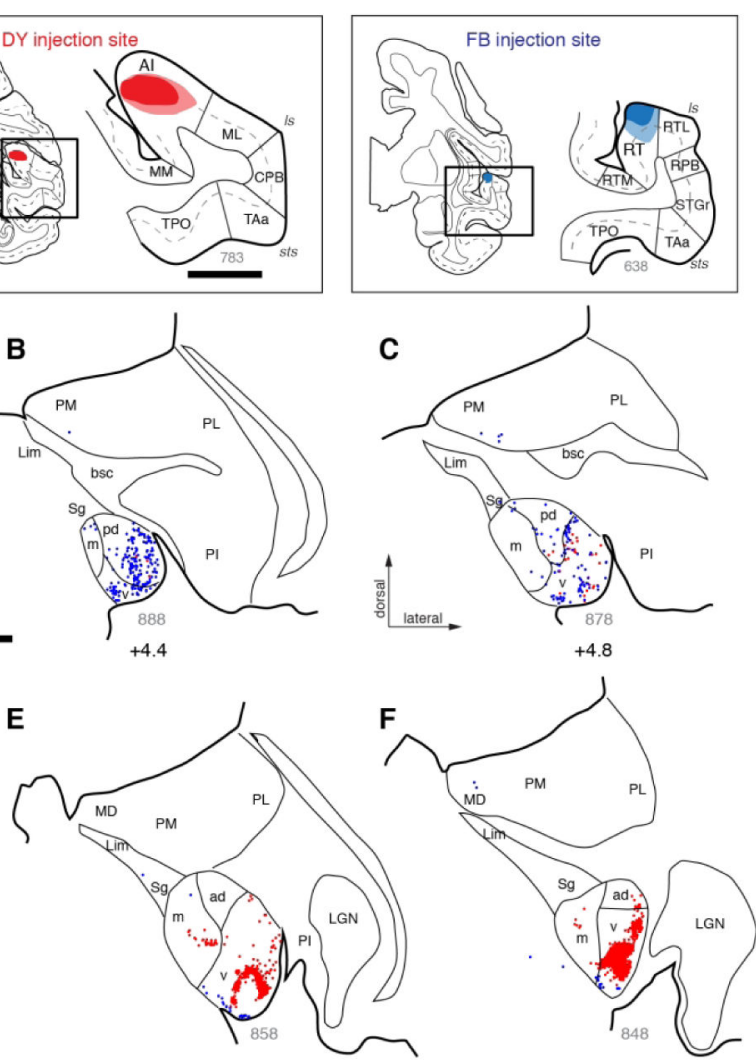

$+5.6$

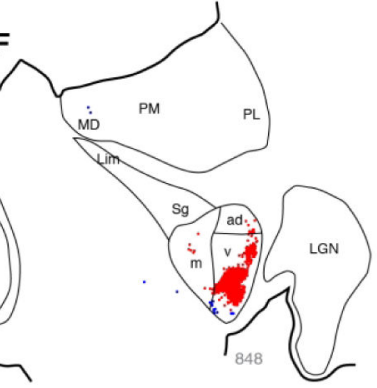

$+6.0$

Figure 4.

Thalamic connections of areas AI and RT in case VA-r. (A-F) Distribution of labeled neurons after injection of retrograde tracers DY into rostral AI (red dots; see Fig. 1B) and FB into caudal RT (blue dots; see Fig. 1G). The injection sites are indicated on coronal histology sections (top center and right), with the superior temporal lobe (in the black rectangles) enlarged on the right. The injection into the caudal core (the rostral part of area AI) labeled neurons in rostral MGN, identifying strong clusters of labeled cells almost exclusively within MGv. The injection into the rostral core (area RT) labeled neurons in the caudal MGN, where more inputs originated from the posterodorsal subdivision (MGpd) than from the ventral subdivision (MGv). Scale bars $=5 \mathrm{~mm}$. For other conventions see Figure 3 . 

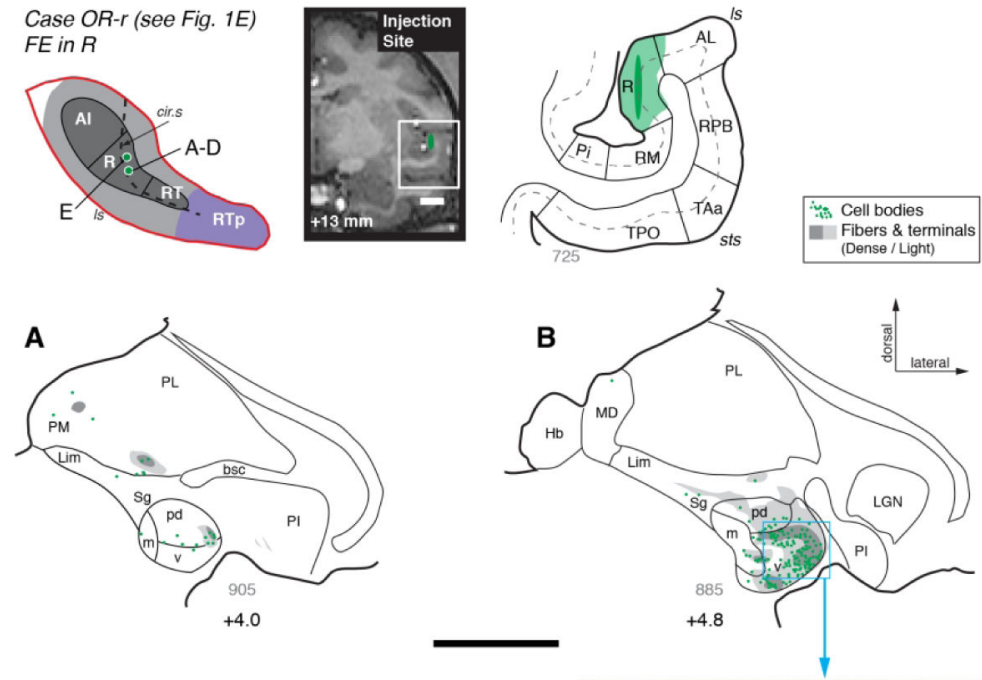

C

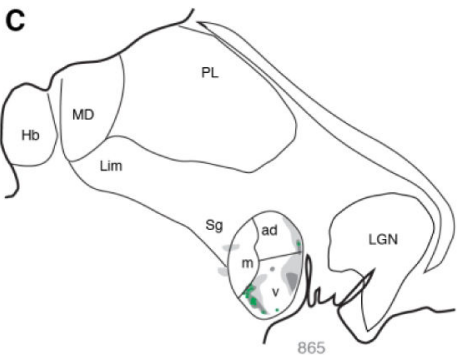

D

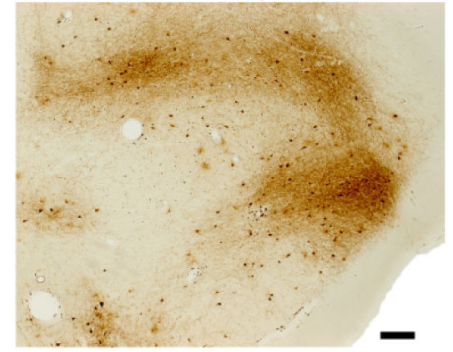

$+5$

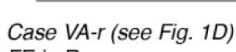

FE in $R$
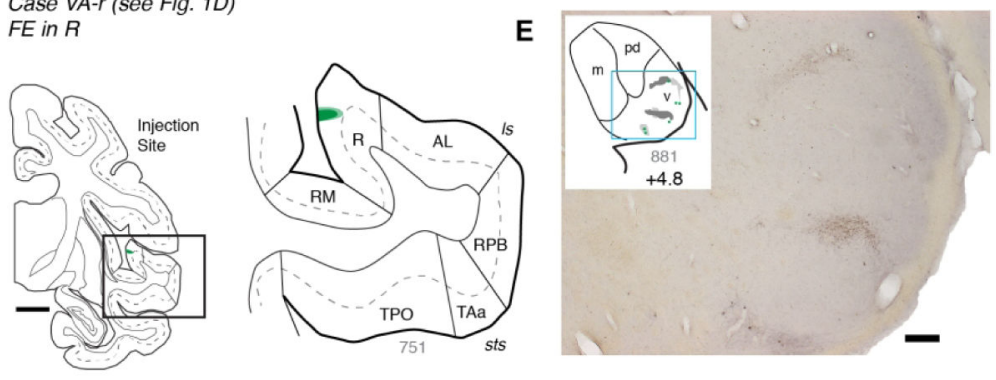

Figure 5.

Thalamic connections of area R in cases OR-r and VA-r. (A-C) Distribution of label after injection of bi-directional tracer FE into area $\mathrm{R}$ in case OR-r. Both retrograde and anterograde label were predominantly found within the MGv, where labeled cells were coextensive with dense patches of labeled terminals and fibers. (D) A photomicrograph of the region outlined by the box in panel B shows the FE positive retrograde and anterograde label in the MGv. (E) Results from a smaller injection of FE restricted to the supragranular layers of area $\mathrm{R}$ in case VA-r. Scattered cells and a few thin fingers of anterograde label were localized to MGv. Scale bars $=0.2 \mathrm{~mm}$ for photomicrographs in D and E, $5 \mathrm{~mm}$ for all other panels. For other conventions see Figure 3. 
Case CC-r (see Fig. 11)

$D Y$ in $R T$
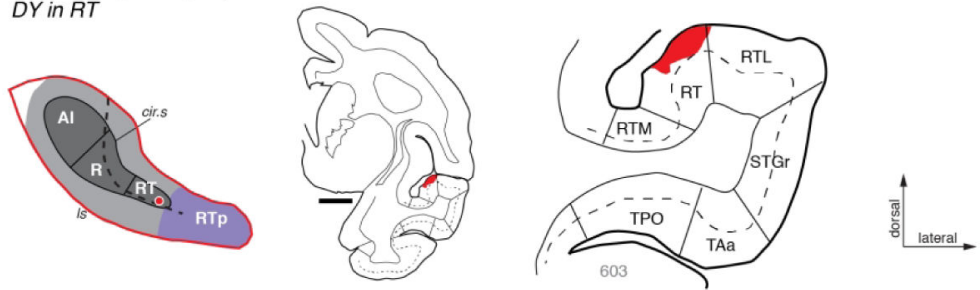

A
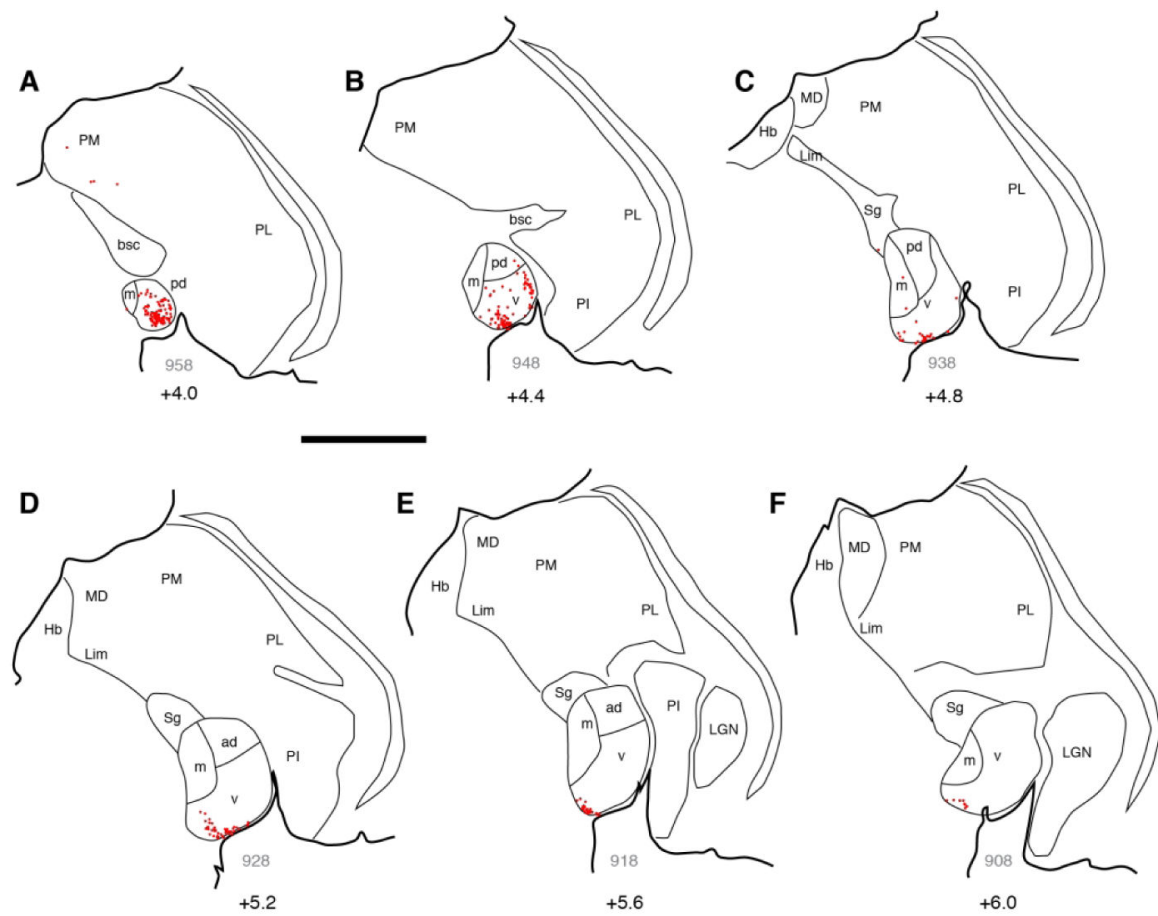

Figure 6.

Thalamic connections of area RT in case CC-r. (A-F) Distribution of labeled neurons after injection of retrograde tracer DY into area RT (see Fig. 1I). Labeled neurons were located primarily in the caudal MGN, including MGpd and MGv. Rostrally, labeled neurons were restricted to the most ventral portion of MGv. The FB injection into RT in another case (Fig. 4) produced a strikingly similar pattern of labeling in the thalamus. Scale bars $=5 \mathrm{~mm}$. For other conventions see Figures 3 and 4. 
Case VA-I (see Fig. 1H) FR in RT (rostral)
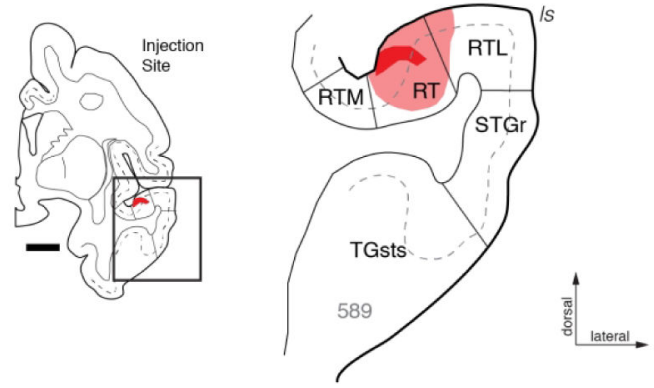

A

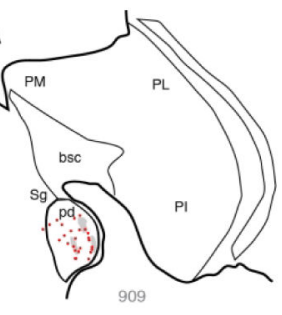

B

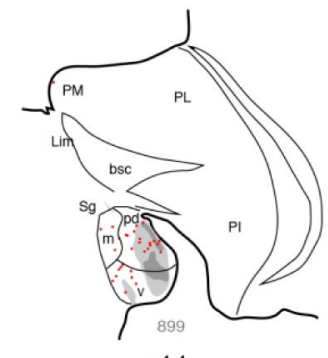

C
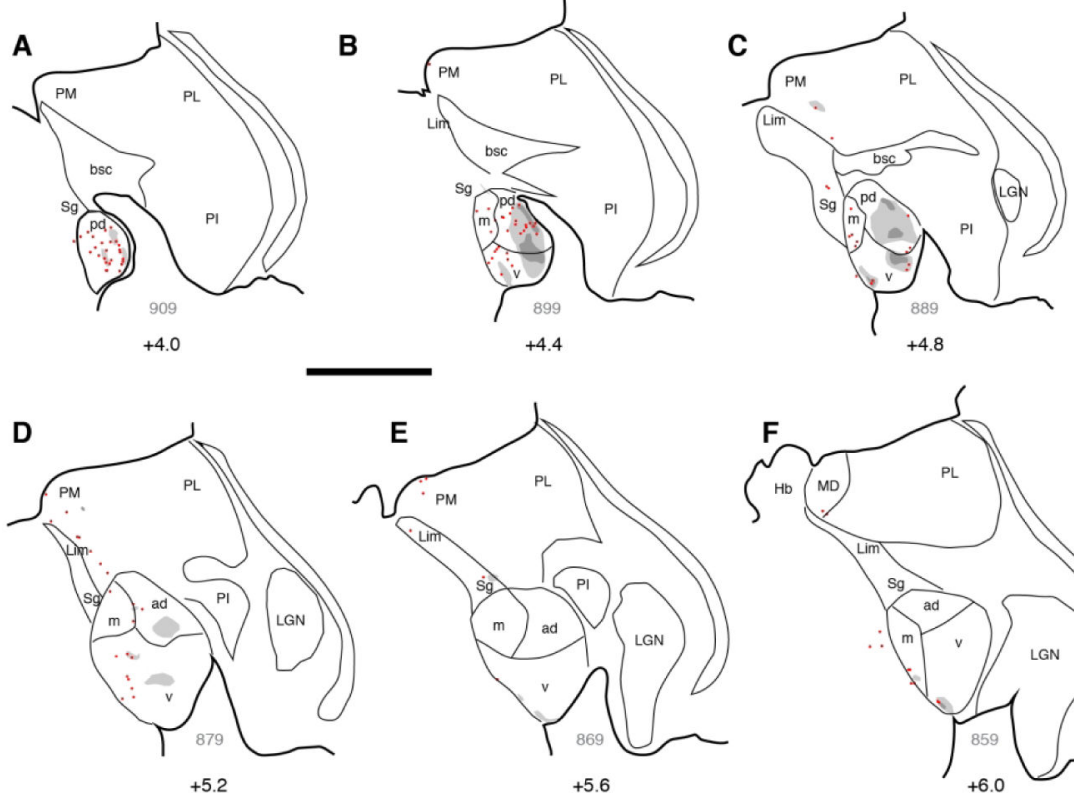

E
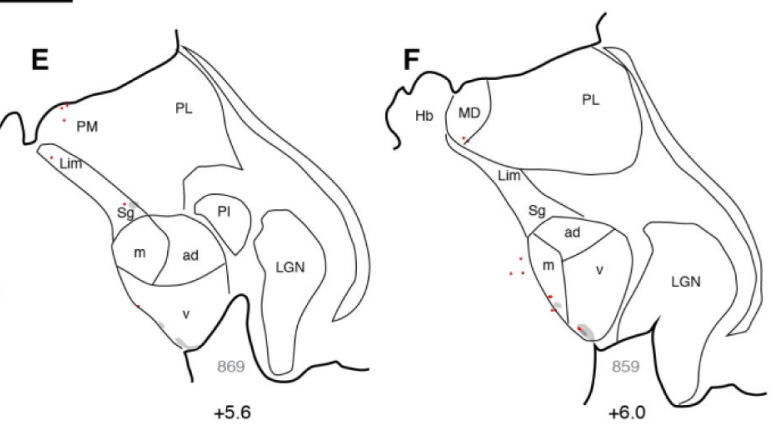

Figure 7.

Thalamic connections of area RT in case VA-1. (A-F) Distribution of retrograde and anterograde label after injection of FR into the rostral end of area RT in case VA-l (see Fig. 1H). As in other RT injections, labeled cells were more numerous in caudal MGN, particularly MGpd, as well as caudal MGv and MGm. Anterograde label was also most extensive within MGpd, though some label was present in MGv (panels B, C). A few labeled cells were scattered dorsomedial to MGm, extending into the medial pulvinar (PM). Scale bars $=5 \mathrm{~mm}$. For other conventions see Figures 3 and 4 . 
Case OR-I (see Fig. 1J) DY in RT/RTp
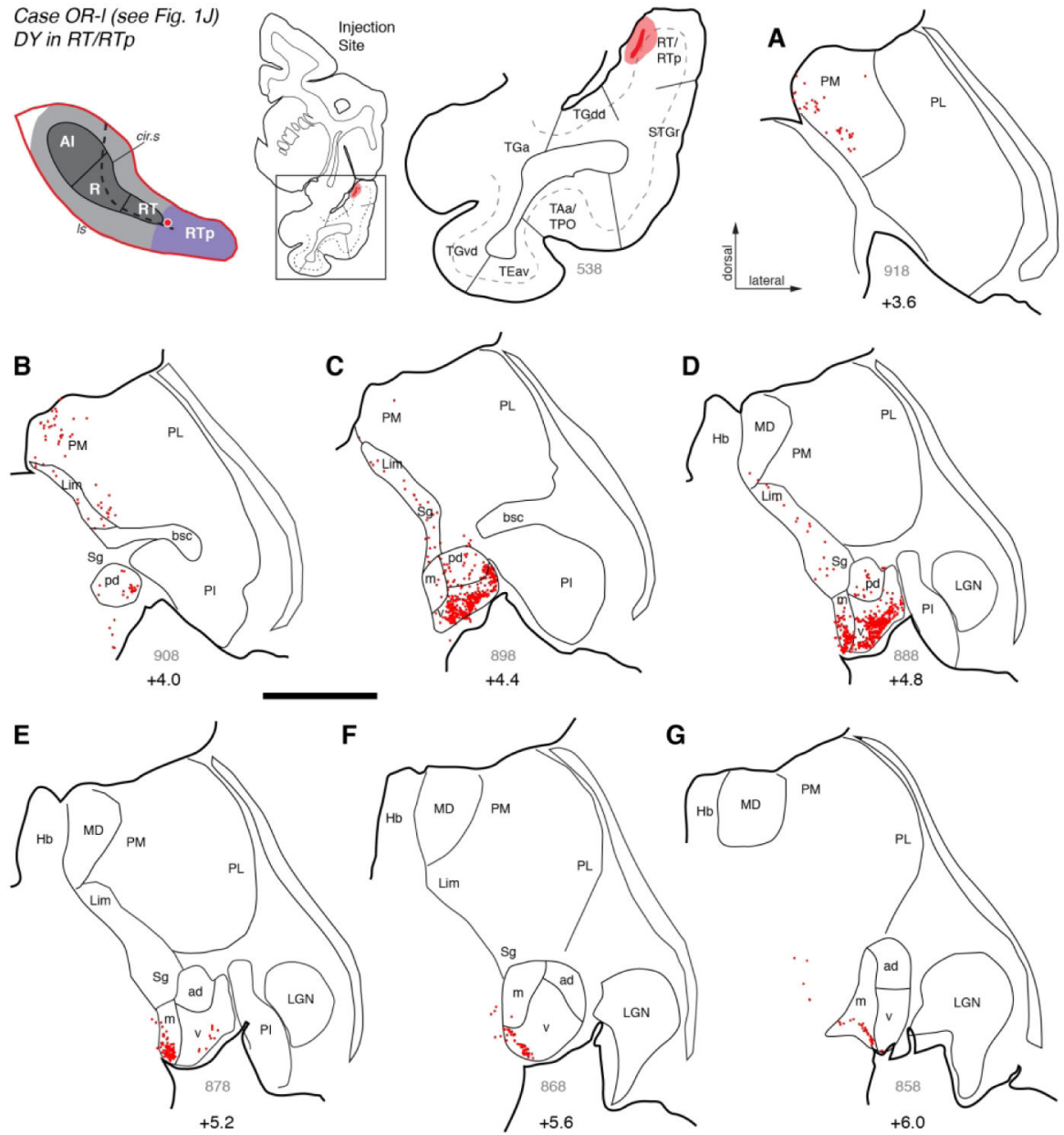

G

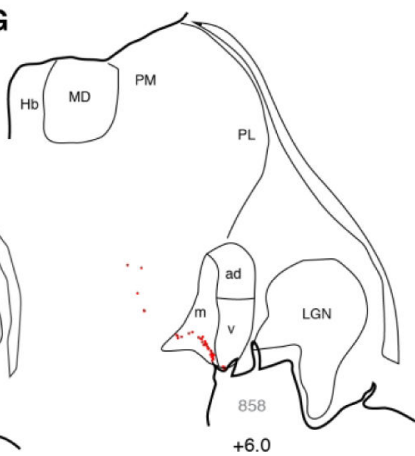

Figure 8.

Thalamic connections of the RT/RTp border in case OR-1. (A-G) Distribution of labeled neurons after injection of retrograde tracer DY into what is most likely caudal RTp, near the border with RT (see Fig. 1J). Labeled neurons were most numerous in the middle portion of MGN, forming a dense cluster in MGv that extended into MGm (panels C-E). Some labeled cells were observed in MGpd (but not in MGad). The few labeled cells in rostral MGN were confined to a narrow strip at the ventral extreme of the nucleus (panels F, G). Two characteristic patterns (labeling in MGpd, and focal labeling in caudal MGv and MGm) are consistent with RT injections. Labeled cells were also observed throughout the $\mathrm{Sg} / \mathrm{Lim}$ nucleus, as well as PM (panels A-C). Scale bars $=5 \mathrm{~mm}$. For other conventions see Figures 3 and 4. 

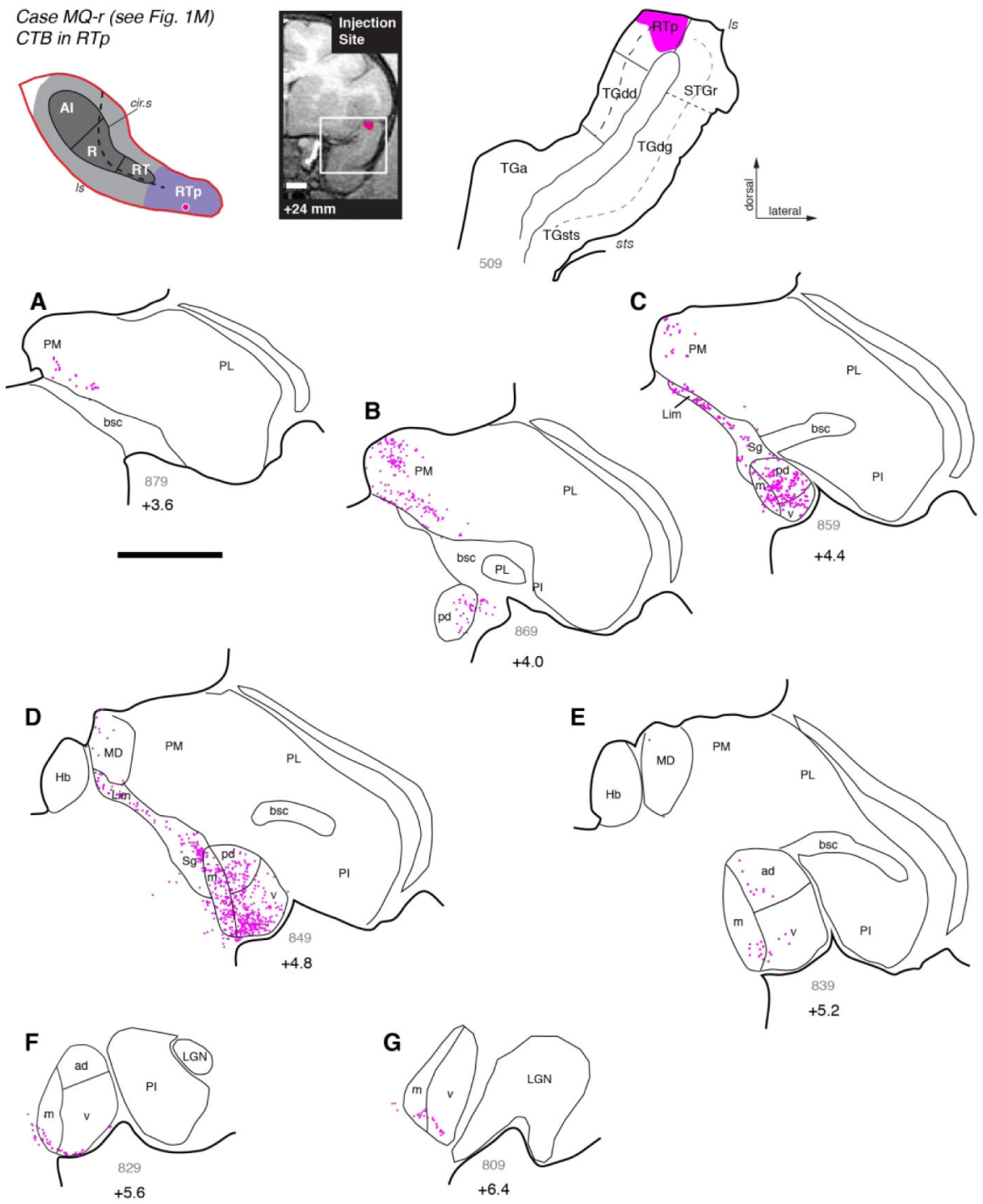

Figure 9.

Thalamic connections of area RTp in case MQ-r. (A-G) Distribution of labeled neurons after injection of retrograde tracer CTB into area RTp, near the border of the STGr (see Fig. 1M). Labeled neurons were located in all subdivisions of the MGN, with a bias toward the posterior half of the nucleus (panels B-D). As was seen after RT injections (Figs. 6 and 8), labeled cells in anterior MGN occupied the ventral edge of MGv and MGm (panels F, G). Relative to RT, a greater proportion of labeled neurons was located outside of the MGN, within the $\mathrm{Sg} / \mathrm{Lim}$ (panels C, D) and PM (panels A, B). Scale bars $=5 \mathrm{~mm}$. For other conventions see Figure 3. 


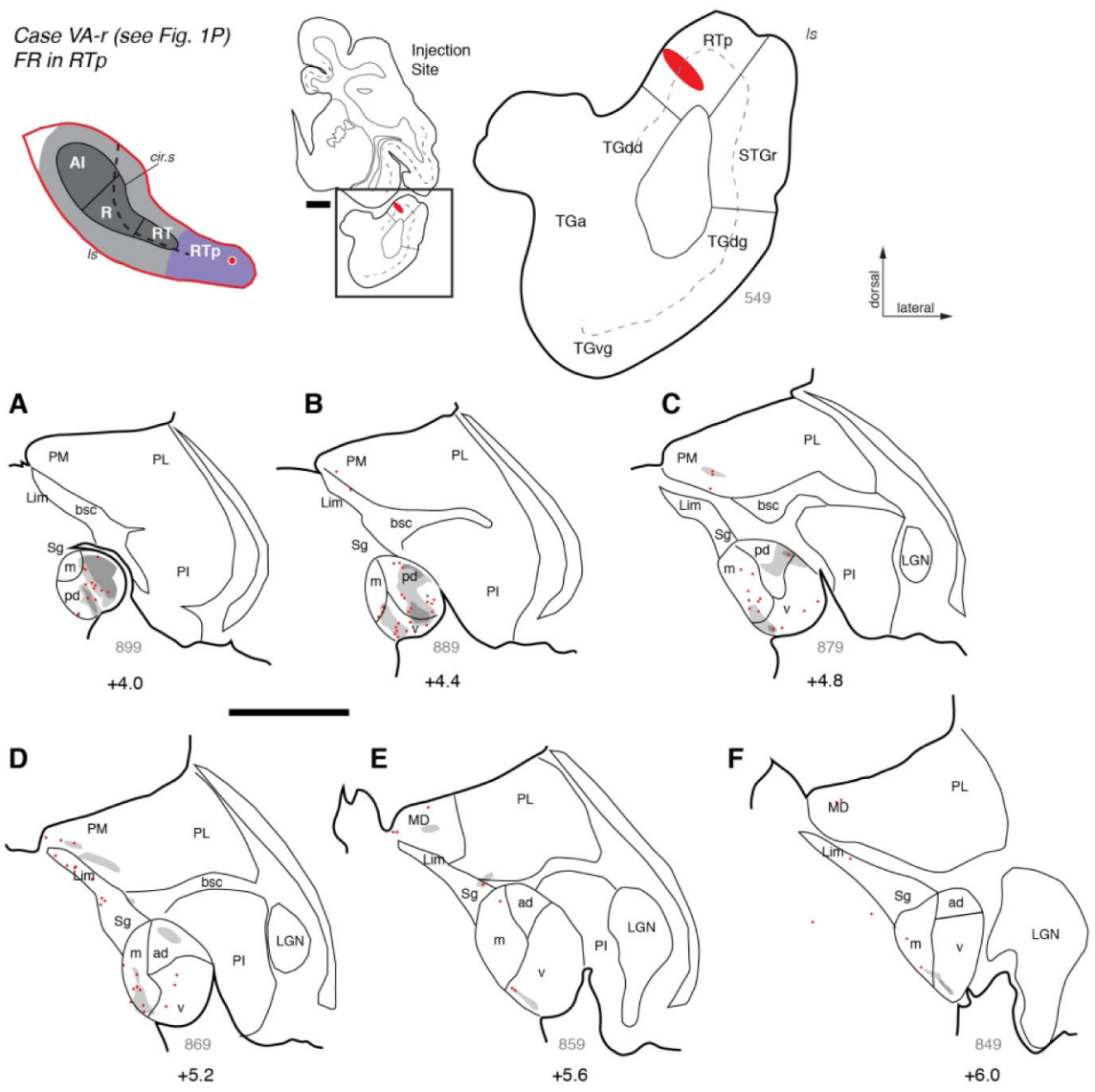

Figure 10.

Thalamic connections of area RTp in case VA-r. (A-F) Distribution of retrograde and anterograde label after injection of bi-directional tracer FR into the rostral portion of area RTp (see Fig. 1P). Both types of label were densest in the posterior MGN, particularly in MGpd (panels A-C), but in the anterior MGN a thin band of anterograde label was located along the ventral edge of MGv (panels E, F). Scale bars $=5 \mathrm{~mm}$. For other conventions see Figures 3 and 4. 
Case OR-I (see Fig. 10) $B D A$ in RTp
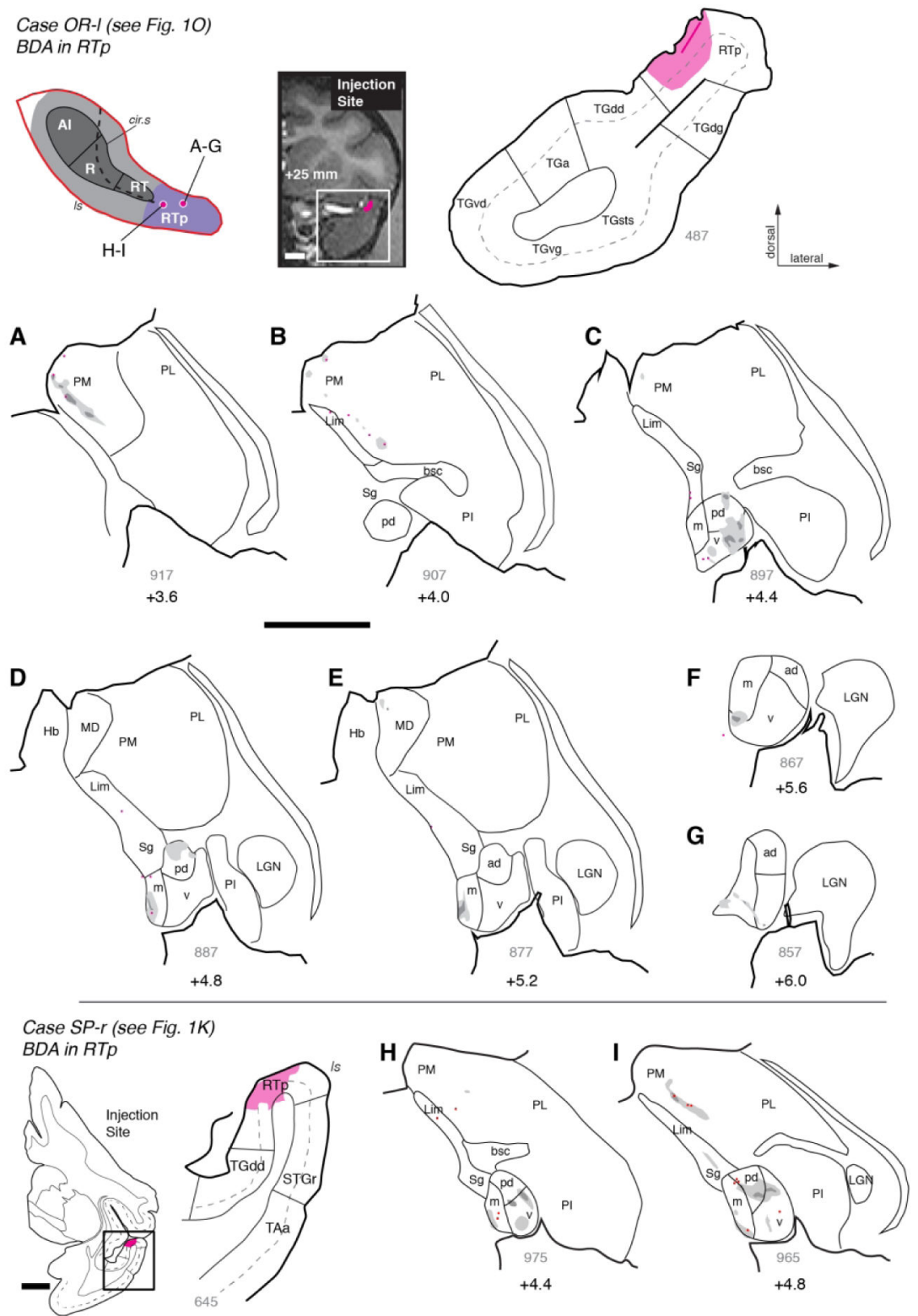

Figure 11.

Corticothalamic connections of area RTp in cases OR-1 and SP-r. (A-G) Distribution of anterograde label (and some retrogradely filled neurons) after injection of BDA into area RTp in case OR-1 (see Fig. 1O). Fibers and terminals were densest in PM (panel A) and MGv (panel C), though some label was located in MGm and MGpd as well. (H, I) Injection of BDA into area RTp in case SP-r (see Fig. 1K) resulted in anterograde label within MGv, MGpd, and MGm, as well as the Sg and PM. Scale bars $=5 \mathrm{~mm}$. For other conventions see Figure 3. 

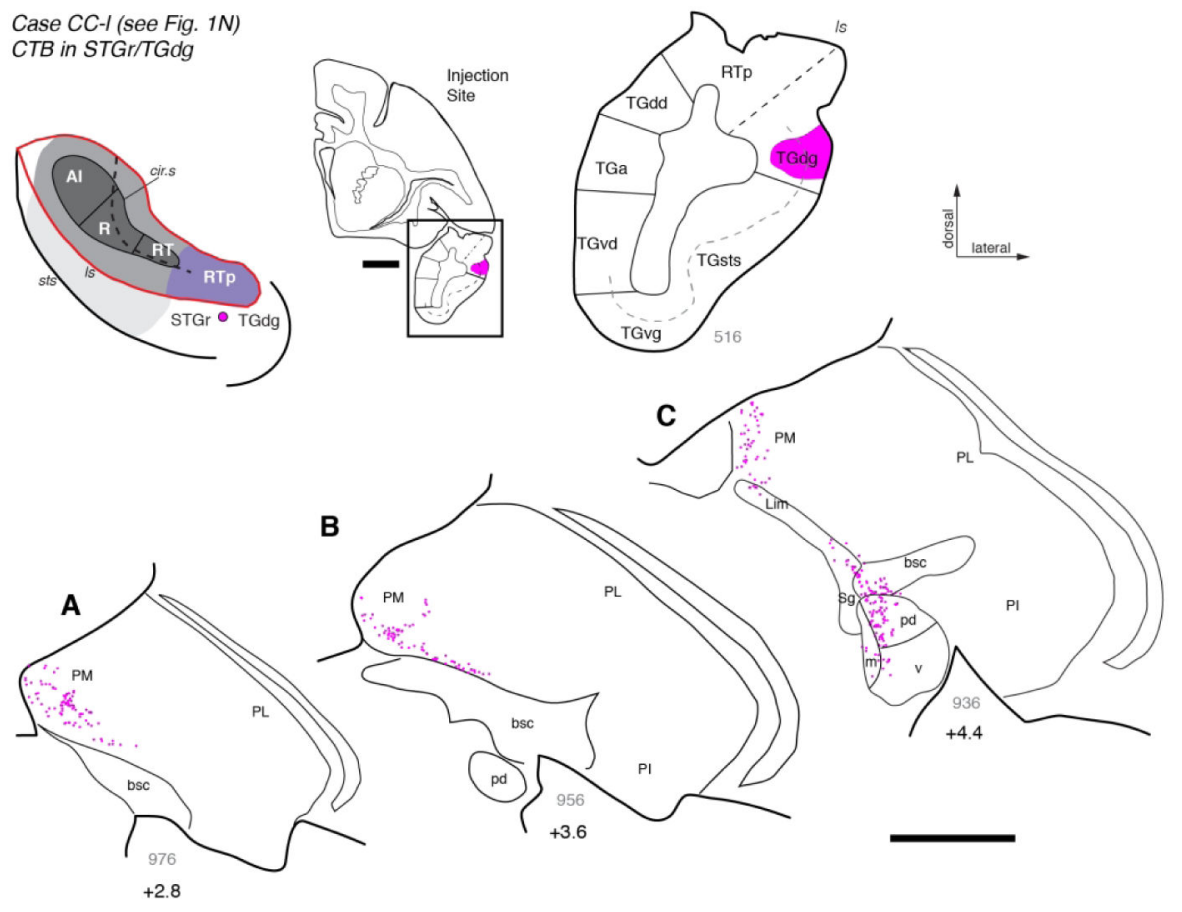

\section{Figure 12.}

Thalamic connections of area TGdg/STGr. (A-C) Distribution of labeled neurons after injection of retrograde tracer CTB into area TGdg, located lateral to RTp on the surface of the rostral STG (see Fig. 1N). Labeled neurons were located throughout the PM, and a continuous cluster spanned the Sg and MGd (panel C). In contradistinction to injections placed on the STP (core and RTp), almost no labeled cells were found within the MGv. Scale bars $=5 \mathrm{~mm}$. For other conventions see Figures 3 and 4 . 
A

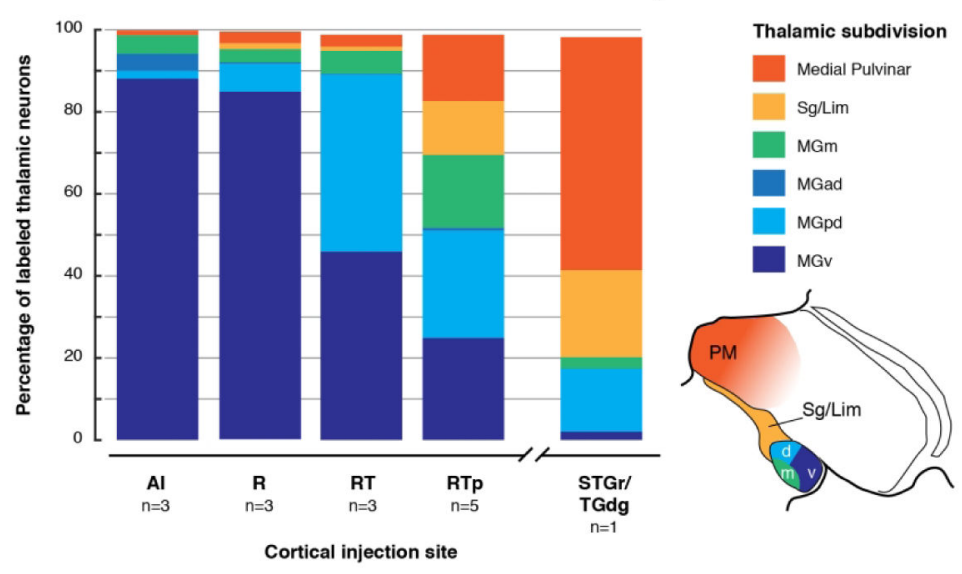

B

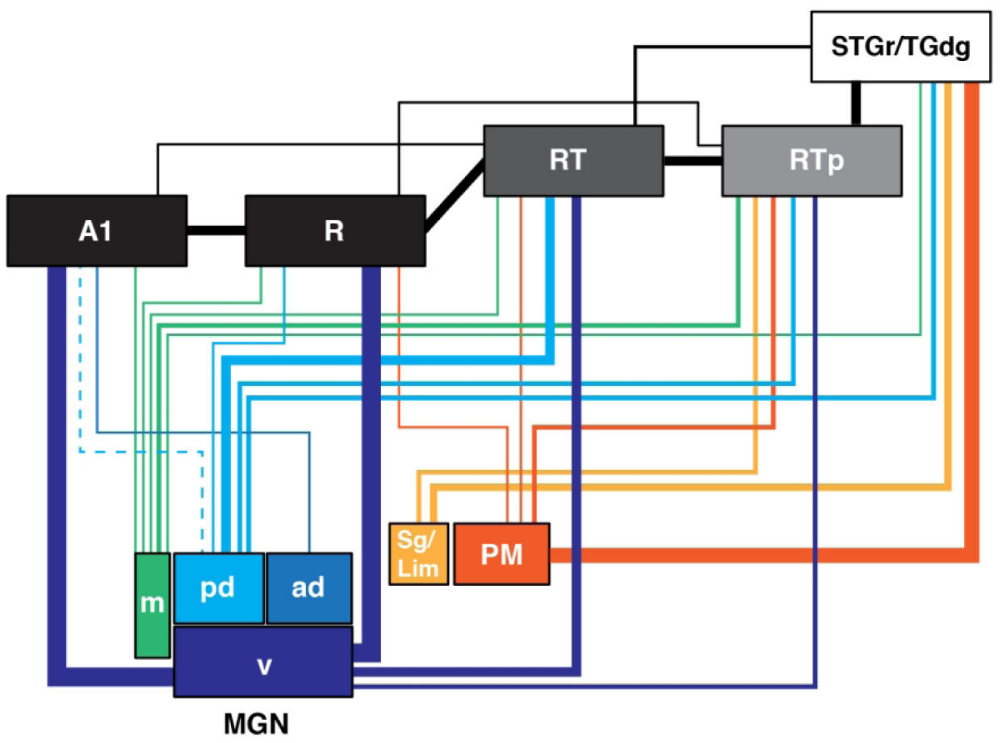

Figure 13.

(A) Distribution of retrogradely labeled neurons in thalamus following injections into the auditory cortical areas of the STP and, for comparison, the STGr. The following cases were combined for each cortical area (see Table 4 for individual injections): AI 1-3; R 4-6; RT 7-9; RTp 11-15; STGr/TGdg, 16. Case 10 was excluded because it could not be designated as RT or RTp with sufficient certainty. Cells in the MD nucleus and those of uncertain location ("other" in Table 4) accounted for $<2 \%$ of labeled cells, and are not pictured. (B) Flowchart diagram of thalamic inputs to the auditory core areas, RTp, and STGr/TGdg. Line thickness corresponds approximately to connection strength, based on the present data. The vertical offsets between AI/R and RT/RTp indicate a feedforward hierarchical relationship suggested by corticocortical connectivity, though all these areas receive direct input from the MGN. There is no significant MGN input to STGr/TGdg, which is placed a higher level than RTp. 


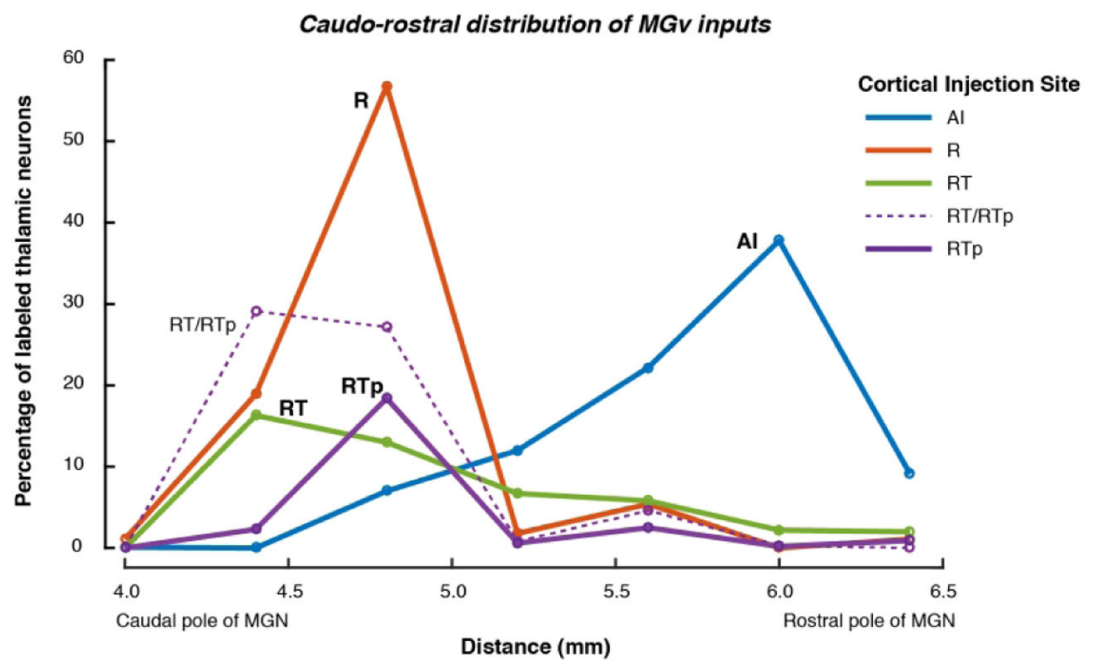

Figure 14.

The distribution of retrogradely labeled cells across the caudal-rostral extent of the $\mathrm{MGV}$ differed across cortical injection sites. Whereas inputs to AI arose from the rostral MGv (blue curve, peaking on the right), inputs to $\mathrm{R}$ were strongly biased toward the caudal $\mathrm{MGV}$ (red curve, peaking on the left). The MGv accounted for a lower overall proportion of inputs to RT and RTp, but those inputs also arose from near the caudal pole of the nucleus. The cases that were combined for each area are the same as in Figure 13A (the injection on the RT/RTp border is plotted individually as a thin dashed line). 
Table 1:

Tracer injections in the supratemporal plane (and STG)

\begin{tabular}{|c|c|c|c|c|c|c|}
\hline Case & Area injected & Tracer & Vol. $(\mu \mathrm{L})$ & Conc. & Supplier \& Catalog \# & Fig. \# \\
\hline 1 (OR-r) & AI (caudal) & FR & 0.5 & $5 \%$ & $\begin{array}{l}\text { Molecular Probes } \\
\text { D-1817/D-3308 }\end{array}$ & $1 \mathrm{~A} ; 3$ \\
\hline 2 (VA-r) & $\mathrm{AI}$ (rostral) & DY & 0.5 & $3 \%$ & Sigma D-0281 & $1 \mathrm{~B} ; 4$ \\
\hline $3(\mathrm{OR}-1)$ & $\mathrm{AI} / \mathrm{R}$ & $\mathrm{FE}$ & 0.5 & $5 \%$ & Mol. Probes D-1820 & $1 \mathrm{C}$ \\
\hline 4 (VA-r) & $\mathrm{R}$ (caudal) & $\mathrm{FE}$ & 0.5 & $5 \%$ & Mol. Probes D-1820 & $1 \mathrm{D} ; 5$ \\
\hline 5 (OR-r) & $\mathrm{R}$ (mid) & $\mathrm{FE}$ & 0.5 & $5 \%$ & Mol. Probes D-1820 & $1 \mathrm{E} ; 5$ \\
\hline $6(\mathrm{VA}-\mathrm{l})$ & $\mathrm{R}$ (rostral) & DY & 0.5 & $3 \%$ & Sigma D-0281 & $1 \mathrm{~F}$ \\
\hline 7 (VA-r) & RT (caudal) & FB & 0.5 & $3 \%$ & Sigma F5756 & $1 \mathrm{G} ; 4$ \\
\hline 8 (VA-1) & RT (rostral) & FR & 0.5 & $5 \%$ & $\begin{array}{l}\text { Molecular Probes } \\
\text { D-1817/D-3308 }\end{array}$ & $1 \mathrm{H} ; 7$ \\
\hline 9 (CC-r) & RT (rostral) & DY & 0.5 & $3 \%$ & Sigma D-0281 & $1 \mathrm{I} ; 6$ \\
\hline $10(\mathrm{OR}-1)$ & $\mathrm{RT} / \mathrm{RTp}$ & DY & 0.3 & $3 \%$ & Sigma D-0281 & $1 \mathrm{~J} ; 8$ \\
\hline 11 (SP-r) & $\mathrm{RTp}$ & BDA & 1.0 & $10 \%$ & Mol. Probes D1956 & $1 \mathrm{~K} ; 11$ \\
\hline $12(\mathrm{CC}-1)$ & $\mathrm{RTp}$ & DY & 0.5 & $3 \%$ & Sigma D-0281 & $1 \mathrm{~L}$ \\
\hline 13 (MQ-r) & RTp (STGr) & СТВ & 1.0 & $2 \%$ & List Biological 103B & $1 \mathrm{M} ; 9$ \\
\hline 14 (OR-1) & $\mathrm{RTp}$ & BDA & 0.5 & $10 \%$ & Mol. Probes D1956 & $10 ; 11$ \\
\hline 15 (VA-r) & $\mathrm{RTp}$ & FR & 0.5 & $5 \%$ & $\begin{array}{l}\text { Molecular Probes } \\
\text { D-1817/D-3308 }\end{array}$ & $1 \mathrm{P} ; 10$ \\
\hline $16(\mathrm{CC}-1)$ & STGr/TGdg & СТВ & 0.5 & $2 \%$ & List Biological 103B & $1 \mathrm{~N} ; 12$ \\
\hline
\end{tabular}


Table 2:

\begin{tabular}{|c|c|c|c|c|c|}
\hline \\
\hline Antibody & $\begin{array}{l}\text { Supplier \& } \\
\text { Catalog \# }\end{array}$ & Type & Host & Dilution & Immunogen \\
\hline Anti-CTB & $\begin{array}{l}\text { List Biological } \\
\# 703\end{array}$ & Polyclonal & Goat & $1: 3200$ & B subunit (choleragenoid) \\
\hline CTB secondary & Vector Labs BA-5000 & $\operatorname{IgG}(\mathrm{H}+\mathrm{L})$ & Rabbit & $1: 50$ & Anti-goat \\
\hline Anti-FR & $\begin{array}{l}\text { Molecular Probes } \\
\text { \#A-6397 }\end{array}$ & $\operatorname{IgG}$ fraction & Rabbit & $1: 4000$ & tetramethylrhodamine \\
\hline Anti-FE & $\begin{array}{l}\text { Molecular Probes } \\
\text { \#A-6413 }\end{array}$ & Polyclonal IgG FAB fragment & Rabbit & $1: 4000$ & fluorescein \\
\hline FR/FE secondary & Vector Labs BA-1000 & $\operatorname{IgG}(\mathrm{H}+\mathrm{L})$ & Goat & $1: 200$ & Anti-rabbit \\
\hline Anti-PV & Sigma \#P3088 & Monoclonal IgG1 & Mouse & $1: 2000$ & PV from purified frog muscle \\
\hline
\end{tabular}


Table of Abbreviations

\begin{tabular}{|c|c|c|}
\hline 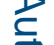 & AI & primary auditory cortex (core) \\
\hline$\overline{\bar{c}}$ & AL & anterolateral belt \\
\hline & bsc & brachium of the superior colliculus \\
\hline & cir.s & circular sulcus \\
\hline & $\mathrm{CL}$ & caudolateral belt \\
\hline ב. & $\mathrm{CM}$ & caudomedial belt \\
\hline & $\mathrm{CPB}$ & caudal parabelt \\
\hline & $\mathrm{Hb}$ & habenula \\
\hline & LGN & lateral geniculate nucleus \\
\hline & ls & lateral sulcus \\
\hline & Lim & limitans nucleus of thalamus \\
\hline & MD & mediodorsal nucleus of thalamus \\
\hline & MGN & medial geniculate nucleus of thalamus \\
\hline & (MG)ad & anterodorsal division of MGN \\
\hline & $(\mathrm{MG}) \mathrm{d}$ & dorsal division of MGN (MGad or MGpd) \\
\hline & $(\mathrm{MG}) \mathrm{m}$ & magnocellular (medial) division of MGN \\
\hline & (MG)pd & posterodorsal division of MGN \\
\hline$\underline{\underline{\rho}}$ & $(\mathrm{MG}) \mathrm{v}$ & ventral division of MGN \\
\hline & ML & middle lateral belt \\
\hline & MM & middle medial belt \\
\hline & $\mathrm{PGa}$ & sts fundus/dorsal bank area \\
\hline & $\mathrm{Pi}$ & parainsular area \\
\hline & PI & inferior pulvinar \\
\hline & PL & lateral pulvinar \\
\hline & PM & medial pulvinar \\
\hline$\overline{\bar{c}}$ & $\mathrm{R}$ & rostral core \\
\hline & Ret & reticular nucleus of thalamus \\
\hline & $\mathrm{RM}$ & rostromedial belt \\
\hline & RPB & rostral parabelt \\
\hline & RT & rostrotemporal core \\
\hline & RTL & rostrotemporal-lateral belt \\
\hline & RTM & rostrotemporal-medial belt \\
\hline & $\mathrm{RTp}$ & rostrotemporal - polar \\
\hline & $\mathrm{Sg}$ & suprageniculate nucleus of thalamus \\
\hline & STGr & rostral superior temporal gyrus \\
\hline & STP & supratemporal plane \\
\hline & sts & superior temporal sulcus \\
\hline$\checkmark$ & STSd & sts dorsal bank \\
\hline & TAa & sts dorsal bank area \\
\hline & $\mathrm{TEa} / \mathrm{TEm}$ & sts ventral bank areas \\
\hline & TEav & ventral subregion of anterior TE \\
\hline
\end{tabular}

J Comp Neurol. Author manuscript; available in PMC 2019 February 11. 
TGa agranular part of the temporal pole

TGdd dysgranular part of the dorsal temporal pole

TGdg granular part of the dorsal temporal pole

TGsts sts part of the temporal pole

TGvd dysgranular part of the ventral temporal pole

TGvg granular part of the ventral temporal pole

TPO sts dorsal bank area

Tpt temporo-parietal area 
Table 4:

Count and proportion of retrogradely labeled thalamic neurons

\begin{tabular}{|c|c|c|c|c|c|c|c|c|c|c|}
\hline Case & Area injected & Tracer & MGv & MGpd & MGad & MGm & sg/lim & PM & MD & other \\
\hline \multirow[t]{2}{*}{1 (OR-r) } & AI (caudal) & FR & 177 & 44 & 9 & 64 & 0 & 31 & 0 & 0 \\
\hline & & & $54.5 \%$ & $13.5 \%$ & $2.8 \%$ & $19.7 \%$ & $0.0 \%$ & $9.5 \%$ & $0.0 \%$ & $0.0 \%$ \\
\hline \multirow[t]{2}{*}{2 (VA-r) } & AI (rostral) & DY & 2112 & 6 & 8 & 56 & 1 & 0 & 0 & 0 \\
\hline & & & $96.7 \%$ & $0.3 \%$ & $0.4 \%$ & $2.6 \%$ & $0.0 \%$ & $0.0 \%$ & $0.0 \%$ & $0.0 \%$ \\
\hline \multirow[t]{2}{*}{$3(\mathrm{OR}-1)$} & $\mathrm{AI} / \mathrm{R}$ & $\mathrm{FE}$ & 165 & 3 & 98 & 5 & 0 & 0 & 0 & 5 \\
\hline & & & $59.8 \%$ & $1.1 \%$ & $35.5 \%$ & $1.8 \%$ & $0.0 \%$ & $0.0 \%$ & $0.0 \%$ & $1.8 \%$ \\
\hline \multirow[t]{2}{*}{4 (VA-r) } & R (caudal) & $\mathrm{FE}$ & 10 & 0 & 0 & 0 & 0 & 0 & 0 & 0 \\
\hline & & & $100.0 \%$ & $0.0 \%$ & $0.0 \%$ & $0.0 \%$ & $0.0 \%$ & $0.0 \%$ & $0.0 \%$ & $0.0 \%$ \\
\hline \multirow[t]{2}{*}{5 (OR-r) } & $\mathrm{R}$ (mid) & $\mathrm{FE}$ & 174 & 18 & 1 & 9 & 4 & 8 & 1 & 0 \\
\hline & & & $80.9 \%$ & $8.4 \%$ & $0.5 \%$ & $4.2 \%$ & $1.9 \%$ & $3.7 \%$ & $0.5 \%$ & $0.0 \%$ \\
\hline \multirow[t]{2}{*}{6 (VA-1) } & $\mathrm{R}$ (rostral) & DY & 54 & 1 & 0 & 0 & 0 & 0 & 0 & 0 \\
\hline & & & $98.2 \%$ & $1.8 \%$ & $0.0 \%$ & $0.0 \%$ & $0.0 \%$ & $0.0 \%$ & $0.0 \%$ & $0.0 \%$ \\
\hline \multirow[t]{2}{*}{7 (VA-r) } & RT (caudal) & FB & 160 & 215 & 0 & 27 & 3 & 7 & 4 & 4 \\
\hline & & & $38.1 \%$ & $51.2 \%$ & $0.0 \%$ & $6.4 \%$ & $0.7 \%$ & $1.7 \%$ & $1.0 \%$ & $1.0 \%$ \\
\hline \multirow[t]{2}{*}{8 (VA-1) } & RT (rostral) & FR & 25 & 45 & 2 & 18 & 4 & 14 & 2 & 0 \\
\hline & & & $22.7 \%$ & $40.9 \%$ & $1.8 \%$ & $16.4 \%$ & $3.6 \%$ & $12.7 \%$ & $1.8 \%$ & $0.0 \%$ \\
\hline \multirow[t]{2}{*}{9 (CC-r) } & RT (rostral) & DY & 209 & 110 & 0 & 3 & 1 & 4 & 0 & 0 \\
\hline & & & $63.9 \%$ & $33.6 \%$ & $0.0 \%$ & $0.9 \%$ & $0.3 \%$ & $1.2 \%$ & $0.0 \%$ & $0.0 \%$ \\
\hline \multirow[t]{2}{*}{10 (OR-1) } & $\mathrm{RT} / \mathrm{RTp}$ & DY & 734 & 73 & 0 & 227 & 63 & 67 & 2 & 17 \\
\hline & & & $62.0 \%$ & $6.2 \%$ & $0.0 \%$ & $19.2 \%$ & $5.3 \%$ & $5.7 \%$ & $0.2 \%$ & $1.4 \%$ \\
\hline \multirow[t]{2}{*}{11 (SP-r) } & $\mathrm{RTp}$ & BDA & 1 & 2 & 0 & 4 & 2 & 4 & 0 & 0 \\
\hline & & & $7.7 \%$ & $15.4 \%$ & $0.0 \%$ & $30.8 \%$ & $15.4 \%$ & $30.8 \%$ & $0.0 \%$ & $0.0 \%$ \\
\hline \multirow[t]{2}{*}{$12(\mathrm{CC}-1)$} & $\mathrm{RTp}$ & DY & 52 & 113 & 4 & 37 & 1 & 27 & 1 & 0 \\
\hline & & & $22.1 \%$ & $48.1 \%$ & $1.7 \%$ & $15.7 \%$ & $0.4 \%$ & $11.5 \%$ & $0.4 \%$ & $0.0 \%$ \\
\hline \multirow[t]{2}{*}{13 (MQ-r) } & RTp (STGr) & СТВ & 332 & 283 & 9 & 219 & 195 & 216 & 12 & 0 \\
\hline & & & $26.2 \%$ & $22.4 \%$ & $0.7 \%$ & $17.3 \%$ & $15.4 \%$ & $17.1 \%$ & $0.9 \%$ & $0.0 \%$ \\
\hline \multirow[t]{2}{*}{14 (OR-1) } & $\mathrm{RTp}$ & BDA & 2 & 0 & 0 & 3 & 5 & 7 & 0 & 0 \\
\hline & & & $11.8 \%$ & $0.0 \%$ & $0.0 \%$ & $17.6 \%$ & $29.4 \%$ & $41.2 \%$ & $0.0 \%$ & $0.0 \%$ \\
\hline \multirow[t]{2}{*}{15 (VA-r) } & RTp & FR & 17 & 26 & 0 & 24 & 11 & 8 & 5 & 1 \\
\hline & & & $18.5 \%$ & $28.3 \%$ & $0.0 \%$ & $26.1 \%$ & $12.0 \%$ & $8.7 \%$ & $5.4 \%$ & $1.1 \%$ \\
\hline \multirow[t]{2}{*}{$16(\mathrm{CC}-1)$} & STGr/TGdg & СТВ & 8 & 61 & 0 & 11 & 84 & 225 & 7 & 0 \\
\hline & & & $2.0 \%$ & $15.4 \%$ & $0.0 \%$ & $2.8 \%$ & $21.2 \%$ & $56.8 \%$ & $1.8 \%$ & $0.0 \%$ \\
\hline
\end{tabular}

